\title{
Dynamical behaviors of a food-chain model with stage structure and time delays
}

\author{
Chuangxia Huang ${ }^{1,2^{*}}$ (D) Yuncheng Qiao ${ }^{1,2}$, Lihong Huang ${ }^{1,2}$ and Ravi P. Agarwal ${ }^{3}$
}

\section{"Correspondence:}

cxiahuang@126.com

'School of Mathematics and

Statistics, Changsha University of

Science and Technology, Changsha, P.R. China

${ }^{2}$ Hunan Key Laboratory of Mathematical Modeling and

Analysis in Engineering, Changsha, P.R. China

Full list of author information is available at the end of the article

\begin{abstract}
Incorporating two delays ( $\tau_{1}$ represents the maturity of predator, $\tau_{2}$ represents the maturity of top predator), we establish a novel delayed three-species food-chain model with stage structure in this paper. By analyzing the characteristic equations, constructing a suitable Lyapunov functional, using Lyapunov-LaSalle's principle, the comparison theorem and iterative technique, we investigate the existence of nonnegative equilibria and their stability. Some interesting findings show that the delays have great impacts on dynamical behaviors for the system: on one hand, if $\tau_{1} \in\left(m_{1}, m_{2}\right)$ and $\tau_{2} \in\left(m_{4},+\infty\right)$, then the boundary equilibrium $E_{2}\left(x^{0}, y_{1}^{0}, y_{2}^{0}, 0,0\right)$ is asymptotically stable (AS), i.e., the prey species and the predator species will coexist, the top-predator species will go extinct; on the other hand, if $\tau_{1} \in\left(m_{2},+\infty\right)$, then the axial equilibrium $E_{1}(k, 0,0,0,0)$ is $A S$, i.e., all predators will go extinct. Numerical simulations are great well agreement with the theoretical results.
\end{abstract}

Keywords: Food-chain model; Stage structure; Time delay; Stability

\section{Introduction}

Predator-prey type interaction is one of basic interspecies relations in the biology and ecology and it is also the basic block of the complicated food chain, food web and biochemical network structure [1-4]. Since the seminal work by Aiello and Freedman [5], species growth models with stage structure have drawn considerable attention (for more details as regards these studies, one can refer to $[6,7])$. Incorporating stage structure for predator into the system, $\mathrm{Xu}$ [8] built a delayed Lotka-Volterra type predator-prey system. Further studies show that the stage structures for both predator and prey should be taken into consideration in modelling [9]. Some interesting results on the dynamical behaviors of predator-prey systems can be found in [10-15].

The 'prey-predator-top-predator' system (the top predator consumes only the predator trophic level), as one of the most important food-chain models [16-21], takes the form

$$
\left\{\begin{array}{l}
\frac{d P_{1}}{d t}=r P_{1}\left(1-\frac{P_{1}}{k}\right)-h_{1} P_{1} P_{2}, \\
\frac{d P_{2}}{d t}=c_{1} P_{1} P_{2}-h_{2} P_{2} T-d_{1} P_{2}, \\
\frac{d T}{d t}=c_{1} P_{2} T-d_{2} T,
\end{array}\right.
$$

where $P_{1}, P_{2}$ and $T$ can be interpreted as the densities of prey species, predator species and top-predator species, respectively. The intrinsic growth rate of the prey species can be

(c) The Author(s) 2018. This article is distributed under the terms of the Creative Commons Attribution 4.0 International License (http://creativecommons.org/licenses/by/4.0/), which permits unrestricted use, distribution, and reproduction in any medium, provided you give appropriate credit to the original author(s) and the source, provide a link to the Creative Commons license, and indicate if changes were made. 
represented as $r . k$ denotes the environmental carrying capacity of the prey species. $h_{1}$ and $h_{2}$ represent the hunting rate of the predator and top predator, respectively. $c_{1}$ and $c_{1}$ can be interpreted as the conversion rate of prey species to its predator species and predator species to the top-predator species, respectively. $d_{1}$ and $d_{2}$ represent the death rate of the predator and top predator, respectively.

A great deal of results on 'prey-predator-top-predator' type food-chain models have been reported in the literature. In [22], the dynamical behaviors of a three-species ratiodependent food-chain model were investigated. Cui et al. [23] discussed the stability and bifurcation of periodic solutions for a three-species food-chain system. Pei et al. [24] established a delay three-species ecosystem with Holling functional response, the dynamical behaviors of the system were studied. In [25], Mbava et al. investigated the dynamics of a food-chain model with disease in species. To a large extent, the existing literature on theoretical studies of 'prey-predator-top-predator' systems is predominantly concerned with cases without stage structure. Literature dealing with the stage structure for both predator and prey appears to be scarce, such studies are, however, important for us to understand the dynamical characteristics of food-chain models. On the other hand, as we know, time delays do exist in many systems, such as population system [26, 27], economic system [28, 29], epidemic model [25, 30], neural network system [31-34], etc. Enlightened by the above discussions, in this paper, we intend to consider a new three-species food-chain model with stage structure and delays for both predator and top predator.

In the following, let us firstly introduce the parameters and a brief sketch of the construction of the model which may indicate the biological relevance of it.

(A1) There are three populations, namely, the prey species whose population density is denoted by $x(t)$, the predator whose immature and mature population densities are $y_{1}(t)$ and $y_{2}(t)$, respectively; the top predator whose immature and mature population densities are described by $z_{1}(t)$ and $z_{2}(t)$, respectively.

(A2) In the absence of predation, the prey population grow according to logistic laws of growth with intrinsic growth rate $\alpha_{1}$, and the carrying capacity is $k$.

(A3) The mature predator consumes the prey with $c_{1} x(t) y_{2}(t)$ and contributes to its immature population growth rate $\alpha_{2} x(t) y_{2}(t)$; the mature top predator consumes the mature predator with $c_{2} y_{2}(t) z_{2}(t)$ and contributes to its immature population growth rate $\alpha_{3} y_{2}(t) z_{2}(t)$.

(A4) The mortality rate of predator is assumed to be proportional to the existing population. We also consider the density dependent mortality rate of the consumer specie as $\beta_{1} y_{2}^{2}(t)$ and $\beta_{2} z_{2}^{2}(t)$. If there is some other factor (other than food) which becomes limiting at high population densities, the self limitation will occur.

According to Table 1 and (A1)-(A4), we can build up the following stage-structured food-chain model:

$$
\left\{\begin{array}{l}
\dot{x}(t)=x(t)\left[\alpha_{1}\left(1-\frac{x(t)}{k}\right)-c_{1} y_{2}(t)\right] \\
\dot{y}_{1}(t)=\alpha_{2} x(t) y_{2}(t)-d_{11} y_{1}(t)-\alpha_{2} e^{-d_{11} \tau_{1}} x\left(t-\tau_{1}\right) y_{2}\left(t-\tau_{1}\right) \\
\dot{y}_{2}(t)=\alpha_{2} e^{-d_{11} \tau_{1}} x\left(t-\tau_{1}\right) y_{2}\left(t-\tau_{1}\right)-d_{12} y_{2}(t)-\beta_{1} y_{2}^{2}(t)-c_{2} y_{2}(t) z_{2}(t) \\
\dot{z}_{1}(t)=\alpha_{3} y_{2}(t) z_{2}(t)-d_{21} z_{1}(t)-\alpha_{3} e^{-d_{21} \tau_{2}} y_{2}\left(t-\tau_{2}\right) z_{2}\left(t-\tau_{2}\right) \\
\dot{z}_{2}(t)=\alpha_{3} e^{-d_{21} \tau_{2}} y_{2}\left(t-\tau_{2}\right) z_{2}\left(t-\tau_{2}\right)-d_{22} z_{2}(t)-\beta_{2} z_{2}^{2}(t)
\end{array}\right.
$$

where all parameters are positive constants. 
Table 1 Parameters for system (2)

\begin{tabular}{ll}
\hline Parameter & Description \\
\hline$\alpha_{1}$ & Intrinsic growth rate of the prey \\
$k$ & Environmental carrying capacity of the prey \\
$c_{1}$ & Capture rate of the mature predator \\
$\frac{\alpha_{2}}{c_{1}}$ & Conversion rate of nutrients into the reproduction of the mature predator \\
$c_{2}$ & Capture rate of the mature top predator \\
$\frac{\alpha_{3}}{c_{2}}$ & Conversion rate of nutrients into the reproduction of the mature top predator \\
$d_{11}$ & Death rate of the immature predator \\
$d_{12}$ & Death rate of the mature predator \\
$d_{21}$ & Death rate of the immature top predator \\
$d_{22}$ & Death rate of the mature top predator \\
$\beta_{1}$ & Intra-specific competition rate of the mature predator species \\
$\beta_{2}$ & Intra-specific competition rate of the mature top-predator species \\
$\tau_{1}$ & Maturity of the predator \\
$\tau_{2}$ & Maturity of the top predator \\
\hline
\end{tabular}

The remainder of this article is organized as follows. In Sect. 2, the preliminaries including the initial conditions, the positivity and boundedness of the solutions of system (2) are presented. In Sect. 3, we deal with the existence of various equilibria. By analyzing the corresponding characteristic equations, the local stability of the equilibria of system (2) are discussed in Sect. 4. In Sect. 5, we investigate the global stability of the interior equilibrium $E^{*}$, the boundary equilibrium $E_{2}$ and the axial equilibrium $E_{1}$. One illustrative example and simulations are shown in Sect. 6. Finally, a brief discussion is drawn in Sect. 7.

\section{Preliminaries}

Considering the biological interpretation of the model, the initial conditions for (2) are required to be

$$
\begin{aligned}
& x(\theta)=\phi(\theta), \quad y_{i}(\theta)=\varphi_{i}(\theta), \quad z_{i}(\theta)=\psi_{i}(\theta), \\
& \phi(0)>0, \quad \varphi_{i}(0)>0, \quad \psi_{i}(0)>0, \quad i=1,2, \quad \theta \in[-\tau, 0],
\end{aligned}
$$

where

$$
\begin{aligned}
& \tau=\max \left\{\tau_{1}, \tau_{2}\right\}, \quad\left(\phi(\cdot), \varphi_{1}(\cdot), \varphi_{2}(\cdot), \psi_{1}(\cdot), \psi_{2}(\cdot)\right) \in C\left([-\tau, 0], R_{+0}^{5}\right), \\
& R_{+0}^{5}=\left\{\left(x_{1}, x_{2}, x_{3}, x_{4}, x_{5}\right): x_{i} \geq 0, i=1,2,3,4,5\right\} .
\end{aligned}
$$

Theorem 1 Let $\Gamma(t)=\left(x(t), y_{1}(t), y_{2}(t), z_{1}(t), z_{2}(t)\right)$ be a solution of system (2) with initial conditions (3), then the solutions of system are strictly positive for all $t \geq 0$.

Proof Firstly, we prioritize $y_{2}(t)$ for $t \in\left[0, \tau^{*}\right]$, where $\tau^{*}=\min \left\{\tau_{1}, \tau_{2}\right\}$. From the initial conditions (3), we can know that $\phi(\theta) \geq 0, \varphi_{2}(\theta) \geq 0$ for $\theta \in[-\tau, 0]$. Thus, we obtain the third equation of system (2), for $t \in\left[0, \tau^{*}\right]$,

$$
\begin{aligned}
\dot{y}_{2}(t) & =\alpha_{2} e^{-d_{11} \tau_{1}} \phi\left(t-\tau_{1}\right) \varphi_{2}\left(t-\tau_{1}\right)-d_{12} y_{2}(t)-\beta_{1} y_{2}^{2}(t)-c_{2} y_{2}(t) z_{2}(t) \\
& \geq-d_{12} y_{2}(t)-\beta_{1} y_{2}^{2}(t)-c_{2} y_{2}(t) z_{2}(t) .
\end{aligned}
$$


By the comparison theorem, we get

$$
y_{2}(t) \geq y_{2}(0) e^{\int_{0}^{t}\left(-d_{12}-\beta_{1} y_{2}(s)-c_{2} z_{2}(s)\right) d s}>0 .
$$

Similarly, from the third equation of system (2), we obtain, for $t \in\left[0, \tau^{*}\right]$,

$$
\begin{aligned}
\dot{z}_{2}(t) & =\alpha_{3} e^{-d_{21} \tau_{2}} \varphi_{2}\left(t-\tau_{2}\right) \psi_{2}\left(t-\tau_{2}\right)-d_{22} z_{2}(t)-\beta_{2} z_{2}^{2}(t) \\
& \geq-d_{22} z_{2}(t)-\beta_{2} z_{2}^{2}(t)
\end{aligned}
$$

since $\varphi_{2}(\theta) \geq 0, \psi_{2}(\theta) \geq 0, \theta \in[-\tau, 0]$.

By the comparison theorem, one has

$$
z_{2}(t) \geq z_{2}(0) e^{\int_{0}^{t}\left(-d_{22}-\beta_{2} z_{2}(s)\right) d s}>0
$$

Repeat the process above, it is obvious to derive that $y_{2}(t)>0, z_{2}(t)>0$ on the intervals $\left[\tau^{*}, 2 \tau^{*}\right], \ldots,\left[n \tau^{*},(n+1) \tau^{*}\right], n \in N$.

The first equation of system (2) together with initial conditions (3) gives

$$
x(t)=x(0) e^{\int_{0}^{t}\left(\alpha_{1}\left(1-\frac{x(s)}{k}\right)-c_{1} y_{2}(s)\right) d s}>0 .
$$

By the second equation of system (2), we can get

$$
y_{1}(t)=\int_{t-\tau_{1}}^{t} \alpha_{2} e^{-d_{11}(t-s)} x(s) y_{2}(s) d s>0
$$

With the fourth equation of system (2), one has

$$
z_{1}(t)=\int_{t-\tau_{2}}^{t} \alpha_{3} e^{-d_{21}(t-s)} y_{2}(s) z_{2}(s) d s>0
$$

This completes the proof.

Remark 1 Taking account for the maturity of predator and top predator, we incorporate two delays in model (2), which is more general than system (1.2) in [8]. To investigate the positivity of system (2), we extend and improve the method in [8]. Specifically, we define a new $\tau^{*}$ satisfying $\tau^{*}=\min \left\{\tau_{1}, \tau_{2}\right\}$. If $t \in\left[0, \tau^{*}\right]$, then $t-\tau_{i} \in[-\tau, 0](i=1,2)$, where $\tau=\max \left\{\tau_{1}, \tau_{2}\right\}$.

Theorem 2 Let $\Gamma(t)=\left(x(t), y_{1}(t), y_{2}(t), z_{1}(t), z_{2}(t)\right)$ be a solution of system (2), then the solutions of system (2) with initial conditions (3) are ultimately bounded.

Proof Define $\rho(t)$ associated with (2) as

$$
\rho(t)=\alpha_{2} x(t)+c_{1} y_{1}(t)+c_{1} y_{2}(t)+\frac{c_{1} c_{2}}{\alpha_{3}} z_{1}(t)+\frac{c_{1} c_{2}}{\alpha_{3}} z_{2}(t)
$$


Denote $d=\min \left\{d_{11}, d_{12}, d_{21}, d_{22}\right\}$, by calculating the derivative of $\rho(t)$ with respect to system (2), we derive

$$
\begin{aligned}
\dot{\rho}(t)= & \alpha_{1} \alpha_{2}\left(1-\frac{x(t)}{k}\right) x(t)-c_{1} d_{11} y_{1}(t)-c_{1}\left(d_{12}+\beta_{1} y_{2}(t)\right) \\
& -\frac{c_{1} c_{2}}{\alpha_{3}} d_{21} z_{1}(t)-\frac{c_{1} c_{2}}{\alpha_{3}}\left(d_{22}+\beta_{2} z_{2}(t)\right) z_{2}(t) \\
\leq & -d \rho(t)+\left(\alpha_{1}+d\right) \alpha_{2} x(t)-\alpha_{1} \alpha_{2} \frac{1}{k} x^{2}(t) \\
\leq & -d \rho(t)+\frac{\alpha_{2} k}{4 \alpha_{1}}\left(\alpha_{1}+d\right)^{2} .
\end{aligned}
$$

Hence, one obtains

$$
\limsup _{t \rightarrow+\infty} \rho(t) \leq \frac{\alpha_{2} k\left(\alpha_{1}+d\right)^{2}}{4 \alpha_{1} d}
$$

This completes the proof.

\section{Existence of equilibria}

In this section, we consider the existence of equilibria. From system (2), $\left(x, y_{1}, y_{2}, z_{1}, z_{2}\right) \in$ $R_{+0}^{5}$ is an equilibrium if and only if:

$$
\left\{\begin{array}{l}
x\left[\alpha_{1}\left(1-\frac{x}{k}\right)-c_{1} y_{2}\right]=0 \\
\alpha_{2} x y_{2}-d_{11} y_{1}-\alpha_{2} e^{-d_{11} \tau_{1}} x y_{2}=0 \\
\alpha_{2} e^{-d_{11} \tau_{1}} x y_{2}-d_{12} y_{2}-\beta_{1} y_{2}^{2}-c_{2} y_{2} z_{2}=0 \\
\alpha_{3} y_{2} z_{2}-d_{21} z_{1}-\alpha_{3} e^{-d_{21} \tau_{2}} y_{2} z_{2}=0 \\
\alpha_{3} e^{-d_{21} \tau_{2}} y_{2} z_{2}-d_{22} z_{2}-\beta_{2} z_{2}^{2}=0
\end{array}\right.
$$

Therefore, there are four equilibria of system (2):

(i) The trivial equilibrium $E_{0}(0,0,0,0,0)$ and the axial equilibrium $E_{1}(k, 0,0,0,0)$ of system (2) exist irrespective of any parametric restriction.

(ii) If the following inequality (C1) holds:

$$
\text { (C1) } \quad \alpha_{2} k e^{-d_{11} \tau_{1}}-d_{12}>0
$$

then there exists the boundary equilibrium boundary equilibrium $E_{2}\left(x^{0}, y_{1}^{0}, y_{2}^{0}, 0,0\right)$, where

$$
\begin{aligned}
& x^{0}=\frac{k\left(\alpha_{1} \beta_{1}+c_{1} d_{12}\right)}{\alpha_{1} \beta_{1}+\alpha_{2} c_{1} k e^{-d_{11} \tau_{1}}}, \\
& y_{1}^{0}=\frac{\alpha_{1} \alpha_{2} k\left(\alpha_{1} \beta_{1}+c_{1} d_{12}\right)\left(1-e^{-d_{11} \tau_{1}}\right)\left(\alpha_{2} k e^{-d_{11} \tau_{1}}-d_{12}\right)}{d_{11}\left(\alpha_{1} \beta_{1}+\alpha_{2} c_{1} k e^{-d_{11} \tau_{1}}\right)^{2}}, \\
& y_{2}^{0}=\frac{\alpha_{1}\left(\alpha_{2} k e^{-d_{11} \tau_{1}}-d_{12}\right)}{\alpha_{1} \beta_{1}+\alpha_{2} c_{1} k e^{-d_{11} \tau_{1}}} .
\end{aligned}
$$


(iii) If the following inequalities (C2), (C3) and (C4) hold:

$$
\begin{aligned}
& \text { (C2) } \alpha_{1} \alpha_{3} c_{2} e^{-d_{21} \tau_{2}}+\beta_{2} c_{1} d_{12}-c_{1} c_{2} d_{22}>0, \\
& \text { (C3) } \alpha_{2} \beta_{2} k e^{-d_{11} \tau_{1}}-\beta_{2} d_{12}+c_{2} d_{22}>0, \\
& \text { (C4) } \alpha_{1} \alpha_{3} e^{-d_{21} \tau_{2}}\left(\alpha_{2} k e^{-d_{11} \tau_{1}}-d_{12}\right)-d_{22}\left(\alpha_{1} \beta_{1}+\alpha_{2} c_{1} k e^{-d_{11} \tau_{1}}\right)>0,
\end{aligned}
$$

then, apart from the axial and boundary equilibria, there exists a unique interior equilibrium $E^{*}\left(x^{*}, y_{1}^{*}, y_{2}^{*}, z_{1}^{*}, z_{2}^{*}\right)$, where

$$
\begin{aligned}
& x^{*}=\frac{k \Lambda_{1}}{\Lambda_{2}}, \quad y_{1}^{*}=\frac{\alpha_{1} \alpha_{2} k\left(1-e^{-d_{11} \tau_{1}}\right)}{d_{11}} \frac{\Lambda_{1} \Lambda_{3}}{\Lambda_{2}^{2}}, \\
& y_{2}^{*}=\frac{\alpha_{1} \Lambda_{3}}{\Lambda_{2}}, \quad z_{1}^{*}=\frac{\alpha_{1} \alpha_{3}\left(1-e^{-d_{21} \tau_{2}}\right)}{d_{21}} \frac{\Lambda_{3} \Lambda_{4}}{\Lambda_{2}^{2}}, \\
& z_{2}^{*}=\frac{\Lambda_{4}}{\Lambda_{2}},
\end{aligned}
$$

and

$$
\begin{aligned}
& \Lambda_{1}=\alpha_{1} \beta_{1} \beta_{2}+\alpha_{1} \alpha_{3} c_{2} e^{-d_{21} \tau_{1}}+\beta_{2} c_{1} d_{12}-c_{1} c_{2} d_{22}, \\
& \Lambda_{2}=\alpha_{1} \beta_{1} \beta_{2}+\alpha_{1} \alpha_{3} c_{2} e^{-d_{21} \tau_{2}}+\alpha_{2} \beta_{2} c_{1} k e^{-d_{11} \tau_{1}}, \\
& \Lambda_{3}=\alpha_{2} \beta_{2} k e^{-d_{11} \tau_{1}}-\beta_{2} d_{12}+c_{2} d_{22}, \\
& \Lambda_{4}=\alpha_{1} \alpha_{3} e^{-d_{21} \tau_{2}}\left(\alpha_{2} k e^{-d_{11} \tau_{1}}-d_{12}\right)-d_{22}\left(\alpha_{1} \beta_{1}+\alpha_{2} c_{1} k e^{-d_{11} \tau_{1}}\right) .
\end{aligned}
$$

Remark 2 Since we consider a three-species-food-chain model, the dynamical behaviors are more complicated and the system has more equilibria than those in [4, 10, 12]. Although these conditions of (C2), (C3) and (C4) seem to be intricate, take Case I (please see the section of Numerical simulation (Sect. 6)) as an example, one can find that these conditions can achieve.

\section{Local stability analysis of the equilibria}

In this section, we study the local stability of system (2) at equilibria. For this purpose, we first introduce the following lemma.

Lemma 1 ([6]) For the equation

$$
\lambda^{2}+a_{1} \lambda+a_{2}+\left(b_{1} \lambda+b_{2}\right) e^{-\lambda \tau}=0
$$

assume that $a_{2}+b_{2} \neq 0, a_{1}^{2}+b_{1}^{2}+b_{2}^{2} \neq 0$, the number of different positive (negative) imaginary roots of (9) can be zero, one, or two only.

If $a_{2}^{2}>b_{2}^{2}$ and $b_{1}^{2}+2 a_{2}-a_{1}^{2}<2 \sqrt{a_{2}^{2}-b_{2}^{2}}$, then (9) (for $\tau>0$ ) has the same stability or instability as when $\tau=0$.

\subsection{The local stability of the trivial equilibrium $E_{0}(0,0,0,0,0)$}

Theorem 3 The trivial equilibrium $E_{0}$ is unstable. 
Proof The characteristic equation for the linearized system of (2) about $E_{0}(0,0,0,0,0)$ is given by

$$
\left|\begin{array}{ccccc}
\lambda-\alpha_{1} & 0 & 0 & 0 & 0 \\
0 & \lambda+d_{11} & 0 & 0 & 0 \\
0 & 0 & \lambda+d_{12} & 0 & 0 \\
0 & 0 & 0 & \lambda+d_{21} & 0 \\
0 & 0 & 0 & 0 & \lambda+d_{22}
\end{array}\right|=0
$$

Then the characteristic equation (10) about the equilibrium $E_{0}$ is

$$
\left(\lambda-\alpha_{1}\right)\left(\lambda+d_{11}\right)\left(\lambda+d_{12}\right)\left(\lambda+d_{21}\right)\left(\lambda+d_{22}\right)=0 .
$$

Since $\lambda_{1}=\alpha_{1}$ is a positive root, the trivial equilibrium $E_{0}(0,0,0,0,0)$ is unstable.

\subsection{The local stability of the axial equilibrium}

Theorem 4 Basing on the existence of equilibria which has been presented in Sect. 3, we have the following results:

(i) If $\alpha_{2} k e^{-d_{11} \tau_{1}}<d_{12}$, then the axial equilibrium $E_{1}$ is locally asymptotically stable $(L A S)$.

(ii) If $\alpha_{2} k e^{-d_{11} \tau_{1}}>d_{12}$, then the axial equilibrium $E_{1}$ is unstable.

Proof The characteristic equation for the linearized system of (2) about $E_{1}(k, 0,0,0,0)$ takes the form

$$
\left|\begin{array}{ccccc}
\lambda+\alpha_{1} & 0 & c_{1} k & 0 & 0 \\
0 & \lambda+d_{11} & \left(e^{-\left(\lambda+d_{11}\right) \tau_{1}}-1\right) \alpha_{2} k & 0 & 0 \\
0 & 0 & \lambda+d_{12}-\alpha_{2} k e^{-d_{11} \tau_{1}} e^{-\lambda \tau_{1}} & 0 & 0 \\
0 & 0 & 0 & \lambda+d_{21} & 0 \\
0 & 0 & 0 & 0 & \lambda+d_{22}
\end{array}\right|=0 .
$$

Hence, the characteristic equation (11) about the equilibrium $E_{1}$ can reduce to

$$
\left(\lambda+\alpha_{1}\right)\left(\lambda+d_{11}\right)\left(\lambda+d_{12}-\alpha_{2} k e^{-d_{11} \tau_{1}} e^{-\lambda \tau_{1}}\right)\left(\lambda+d_{21}\right)\left(\lambda+d_{22}\right)=0 .
$$

It is obvious that $\lambda_{1}=-\alpha_{1}, \lambda_{2}=-d_{11}, \lambda_{3}=-d_{21}, \lambda_{4}=-d_{22}$ are all negative eigenvalues, thus the stability of axial equilibrium $E_{1}$ is determined by the equation of $\lambda+d_{12}-$ $\alpha_{2} k e^{-d_{11} \tau_{1}} e^{-\lambda \tau_{1}}=0$. Let $f(\lambda)$ have the following form:

$$
f(\lambda)=\lambda+d_{12}-\alpha_{2} k e^{-d_{11} \tau_{1}} e^{-\lambda \tau_{1}} .
$$

By analyzing, one can obtain the following cases.

If $\alpha_{2} k e^{-d_{11} \tau_{1}}<d_{12}$, we assume that $\operatorname{Re} \lambda \geq 0$. By calculating, we get

$$
\begin{aligned}
\operatorname{Re} \lambda & =-d_{12}+\alpha_{2} k e^{-d_{11} \tau_{1}} e^{-\tau_{1} \operatorname{Re} \lambda} \cos \left(\tau_{1} \operatorname{Im} \lambda\right) \\
& \leq-d_{12}+\alpha_{2} k e^{-d_{11} \tau_{1}}<0,
\end{aligned}
$$

which is a contradiction. Hence, $\operatorname{Re} \lambda<0$. Consequently, the result (i) of Theorem 4 holds. 
If $\alpha_{2} k e^{-d_{11} \tau_{1}}>d_{12}$, then we have

$$
\left\{\begin{array}{l}
f(0)=d_{12}-\alpha_{2} k e^{-d_{11} \tau_{1}}<0, \\
\lim _{\lambda \rightarrow+\infty} f(\lambda)=+\infty, \\
f^{\prime}(\lambda)=1+\tau_{1} \alpha_{2} k e^{-d_{11} \tau_{1}} e^{-\lambda \tau_{1}}>0 .
\end{array}\right.
$$

Therefore, for $f(\lambda)=0$ there must exist a positive root. Thus, the result (ii) of Theorem 4 holds as well. This completes the proof.

\subsection{The local stability of the boundary equilibrium $E_{2}\left(x^{0}, y_{1}^{0}, y_{2}^{0}, 0,0\right)$}

Theorem 5 Under the condition (C1), we get the following results:

(i) If $\alpha_{3} y_{2}^{0} e^{-d_{21} \tau_{2}}<d_{22}$ and $\alpha_{1} \beta_{1}-\alpha_{2} c_{1} k e^{-d_{11} \tau_{1}}>0$, then the boundary equilibrium $E_{2}$ is LAS.

(ii) If $\alpha_{3} y_{2}^{0} e^{-d_{21} \tau_{2}}>d_{22}$, then the boundary equilibrium $E_{2}$ is unstable.

Proof The characteristic equation for the linearized system of (2) about $E_{2}\left(x^{0}, y_{1}^{0}, y_{2}^{0}, 0,0\right)$ is given as below

$$
\left|\begin{array}{ccccc}
\lambda+\frac{\alpha_{1} x^{0}}{k} & 0 & c_{1} x^{0} & 0 & 0 \\
\alpha_{2} \Delta_{3} y_{2}^{0} & \lambda+d_{11} & \alpha_{2} \Delta_{3} x^{0} & 0 & 0 \\
-\alpha_{2} e^{-\left(\lambda+d_{11} \tau_{1}\right.} y_{2}^{0} & 0 & \lambda+\Delta_{1} & 0 & c_{2} y_{2}^{0} \\
0 & 0 & 0 & \lambda+d_{21} & \alpha_{3} \Delta_{4} y_{2}^{0} \\
0 & 0 & 0 & 0 & \lambda+\Delta_{2}
\end{array}\right|=0
$$

where

$$
\begin{aligned}
& \Delta_{1}=\beta_{1} y_{2}^{0}+\alpha_{2} x^{0} e^{-d_{11} \tau_{1}}\left(1-e^{-\lambda \tau_{1}}\right), \\
& \Delta_{2}=d_{22}-\alpha_{3} y_{2}^{0} e^{-d_{21} \tau_{2}} e^{-\lambda \tau_{2}}, \\
& \Delta_{3}=e^{-\left(\lambda+d_{11}\right) \tau_{1}}-1, \\
& \Delta_{4}=e^{-\left(\lambda+d_{21}\right) \tau_{2}}-1 .
\end{aligned}
$$

Thus, the characteristic equation (12) about the equilibrium $E_{2}$ is

$$
\left(\lambda+d_{11}\right)\left(\lambda+d_{21}\right)\left(\lambda+\Delta_{2}\right)\left[\left(\lambda+\Delta_{1}\right)\left(\lambda+\frac{\alpha_{1} x^{0}}{k}\right)+c_{1} \alpha_{2} x^{0} y_{2}^{0} e^{-\left(\lambda+d_{11}\right) \tau_{1}}\right]=0 .
$$

Clearly, $\lambda_{1}=-d_{11}, \lambda_{2}=-d_{21}$, which are always negative. Hence, the stability of the boundary equilibrium $E_{2}$ is determined by the following equations:

$$
\lambda+\Delta_{2}=0,
$$

and

$$
\left(\lambda+\Delta_{1}\right)\left(\lambda+\frac{\alpha_{1} x^{0}}{k}\right)+c_{1} \alpha_{2} x^{0} y_{2}^{0} e^{-\left(\lambda+d_{11}\right) \tau_{1}}=0
$$


For $\lambda+\Delta_{2}=0$, that is, $\lambda+d_{22}-\alpha_{3} y_{2}^{0} e^{-d_{21} \tau_{2}} e^{-\lambda \tau_{2}}=0$, let $f(\lambda)$ have the following form:

$$
f(\lambda)=\lambda+d_{22}-\alpha_{3} y_{2}^{0} e^{-d_{21} \tau_{2}} e^{-\lambda \tau_{2}}
$$

By analyzing, one can obtain the following cases.

If $\alpha_{3} y_{2}^{0} e^{-d_{21} \tau_{2}}>d_{22}$, then we have

$$
\left\{\begin{array}{l}
f(0)=d_{22}-\alpha_{3} y_{2}^{0} e^{-d_{21} \tau_{2}}<0 \\
\lim _{\lambda \rightarrow+\infty} f(\lambda)=+\infty \\
f^{\prime}(\lambda)=1+\tau_{2} \alpha_{3} y_{2}^{0} e^{-d_{21} \tau_{2}} e^{-\lambda \tau_{2}}>0
\end{array}\right.
$$

Thus, for $f(\lambda)=0$ there must exist a positive root, thereby, the result (ii) of Theorem 5 holds.

If $\alpha_{3} y_{2}^{0} e^{-d_{21} \tau_{2}}<d_{22}$, we assume that $\operatorname{Re} \lambda \geq 0$. By calculating, we get

$$
\begin{aligned}
\operatorname{Re} \lambda & =-d_{22}+\alpha_{3} y_{2}^{0} e^{-d_{21} \tau_{2}} e^{-\tau_{2} \operatorname{Re} \lambda} \cos \left(\tau_{2} \operatorname{Im} \lambda\right) \\
& \leq-d_{22}+\alpha_{3} y_{2}^{0} e^{-d_{21} \tau_{2}}<0
\end{aligned}
$$

which is a contradiction. Hence, $\operatorname{Re} \lambda<0$.

For $\left(\lambda+\Delta_{1}\right)\left(\lambda+\frac{\alpha_{1} x^{0}}{k}\right)+c_{1} \alpha_{2} x^{0} y_{2}^{0} e^{-\left(\lambda+d_{11}\right) \tau_{1}}=0$, by calculating, we can obtain

$$
\lambda^{2}+a_{1} \lambda+a_{2}+\left(b_{1} \lambda+b_{2}\right) e^{-\lambda \tau_{1}}=0
$$

where

$$
\begin{aligned}
& a_{1}=\frac{\alpha_{1} x^{0}}{k}+\beta_{1} y_{2}^{0}+\alpha_{2} x^{0} e^{-d_{11} \tau_{1}}, \quad a_{2}=\frac{\alpha_{1} x^{0}}{k}\left(\beta_{1} y_{2}^{0}+\alpha_{2} x^{0} e^{-d_{11} \tau_{1}}\right), \\
& b_{1}=-\alpha_{2} x^{0} e^{-d_{11} \tau_{1}}, \quad b_{2}=\alpha_{2} x^{0} e^{-d_{11} \tau_{1}}\left(c_{1} y_{2}^{0}-\frac{\alpha_{1} x^{0}}{k}\right) .
\end{aligned}
$$

When $\tau_{1}=0$, Eq. (13) can reduce to

$$
\lambda^{2}+\left(\frac{\alpha_{1} x^{0}}{k}+\beta_{1} y_{2}^{0}\right) \lambda+x^{0} y_{2}^{0}\left(\frac{\alpha_{1} \beta_{1}}{k}+\alpha_{2} c_{1}\right)=0
$$

Obviously, there only exist negative eigenvalues. Hence, the boundary equilibrium $E_{2}$ is LAS when $\tau_{1}=0$ and $\alpha_{3} y_{2}^{0} e^{-d_{21} \tau_{2}}<d_{22}$.

When $\tau_{1} \neq 0$, one can derive that

$$
b_{1}^{2}+2 a_{2}-a_{1}^{2}=-\left(\frac{\alpha_{1} x^{0}}{k}\right)^{2}-\left(\beta_{1} y_{2}^{0}\right)^{2}-2 \alpha_{2} \beta_{1} x^{0} y_{2}^{0} e^{-d_{11} \tau_{1}}<0,
$$

and

$$
\begin{aligned}
a_{2}^{2}-b_{2}^{2}= & \frac{\left(x^{0}\right)^{2} y_{2}^{0}}{k^{2}}\left[2 k \alpha_{1} \alpha_{2} e^{-d_{11} \tau_{1}}\left(\alpha_{1} \beta_{1}+c_{1} d_{12}\right)\right. \\
& \left.+\left(\alpha_{1} \beta_{1}-\alpha_{2} c_{1} k e^{-d_{11} \tau_{1}}\right) \alpha_{1}\left(\alpha_{2} k e^{-d_{11} \tau_{1}}-d_{12}\right)\right] .
\end{aligned}
$$


Under the condition (C1) $\alpha_{2} k e^{-d_{11} \tau_{1}}-d_{12}>0$, if $\alpha_{1} \beta_{1}-\alpha_{2} c_{1} k e^{-d_{11} \tau_{1}}>0$, then $a_{2}^{2}>b_{2}^{2}$, by Lemma 1 , the boundary equilibrium $E_{2}$ is LAS. Therefore, the result (i) of Theorem 5 holds as well. This completes the proof.

\subsection{The stability of the interior equilibrium $E^{*}\left(x^{*}, y_{1}^{*}, y_{2}^{*}, z_{1}^{*}, z_{2}^{*}\right)$}

Theorem 6 Under the conditions (C2), (C3) and (C4), if $2 \alpha_{1}>\alpha_{2} k e^{-d_{11} \tau_{1}}, 2 \beta_{1}>\alpha_{2} e^{-d_{11} \tau_{1}}+$ $\alpha_{3} e^{-d_{21} \tau_{2}}$ and $2 \beta_{2}>\alpha_{3} e^{-d_{21} \tau_{2}}$, then the interior equilibrium $E^{*}$ is stable.

Proof The linearized system of (2) about $E^{*}\left(x^{*}, y_{1}^{*}, y_{2}^{*}, z_{1}^{*}, z_{2}^{*}\right)$ is

$$
\left\{\begin{aligned}
\dot{x}(t)= & -\frac{\alpha_{1}}{k} x^{*} x(t)-c_{1} x^{*} y_{2}(t) \\
\dot{y}_{1}(t)= & \alpha_{2} y_{2}^{*} x(t)+\alpha_{2} x^{*} y_{2}(t)-d_{11} y_{1}(t)-\alpha_{2} e^{-d_{11} \tau_{1}} y_{2}^{*} x\left(t-\tau_{1}\right) \\
& -\alpha_{2} e^{-d_{11} \tau_{1}} x^{*} y_{2}\left(t-\tau_{1}\right) \\
\dot{y}_{2}(t)= & \alpha_{2} e^{-d_{11} \tau_{1}} y_{2}^{*} x\left(t-\tau_{1}\right)+\alpha_{2} e^{-d_{11} \tau_{1}} x^{*} y_{2}\left(t-\tau_{1}\right) \\
& -\left(\alpha_{2} e^{-d_{11} \tau_{1}} x^{*}+\beta_{1} y_{2}^{*}\right) y_{2}(t)-c_{2} y_{2}^{*} z_{2}(t) \\
\dot{z}_{1}(t)= & \alpha_{3} z_{2}^{*} y_{2}(t)+\alpha_{3} y_{2}^{*} z_{2}(t)-d_{21} z_{1}(t)-\alpha_{3} e^{-d_{21} \tau_{2}} z_{2}^{*} y_{2}\left(t-\tau_{2}\right) \\
& -\alpha_{3} e^{-d_{21} \tau_{2}} y_{2}^{*} z_{2}\left(t-\tau_{2}\right) \\
\dot{z}_{2}(t)= & \alpha_{3} e^{-d_{21} \tau_{2}} z_{2}^{*} y_{2}\left(t-\tau_{2}\right)+\alpha_{3} e^{-d_{21} \tau_{2}} y_{2}^{*} z_{2}\left(t-\tau_{2}\right)-\left(\alpha_{3} e^{-d_{21} \tau_{2}} y_{2}^{*}+\beta_{2} z_{2}^{*}\right) z_{2}^{2}(t)
\end{aligned}\right.
$$

Define $V\left(x_{t}, y_{1 t}, y_{2 t}, z_{1 t}, z_{2 t}\right)$ associated with (14) as

$$
\begin{aligned}
V\left(x_{t}, y_{1 t}, y_{2 t}, z_{1 t}, z_{1 t}\right)= & \frac{1}{2 x^{*}} x^{2}(t)+\frac{1}{2 y_{2}^{*}} y_{2}^{2}(t)+\frac{1}{2 z_{2}^{*}} z_{2}^{2}(t) \\
& +\frac{x^{*}}{2 y_{2}^{*}} \alpha_{2} e^{-d_{11} \tau_{1}} \int_{t-\tau_{1}}^{t} y_{2}^{2}(s) d s+\frac{y_{2}^{*}}{2 z_{2}^{*}} \alpha_{3} e^{-d_{21} \tau_{2}} \int_{t-\tau_{2}}^{t} z_{2}^{2}(s) d s \\
& +\frac{\alpha_{2} e^{-d_{11} \tau_{1}}}{2} \int_{t-\tau_{1}}^{t} x^{2}(s) d s+\frac{\alpha_{3} e^{-d_{21} \tau_{2}}}{2} \int_{t-\tau_{2}}^{t} y_{2}^{2}(s) d s .
\end{aligned}
$$

By calculating the derivative of $V\left(x_{t}, y_{1 t}, y_{2 t}, z_{1 t}, z_{2 t}\right)$ with respect to system (14), we derive

$$
\begin{aligned}
\dot{V}\left(x_{t}, y_{1 t}, y_{2 t}, z_{1 t}, z_{2 t}\right)= & \frac{1}{x^{*}} x(t) \dot{x}(t)+\frac{1}{y_{2}^{*}} y_{2}(t) \dot{y}_{2}(t)+\frac{1}{z_{2}^{*}} z_{2}(t) \dot{z}_{2}(t) \\
& +\frac{x^{*}}{2 y_{2}^{*}} \alpha_{2} e^{-d_{11} \tau_{1}}\left[y_{2}^{2}(t)-y_{2}^{2}\left(t-\tau_{1}\right)\right] \\
& +\frac{y_{2}^{*}}{2 z_{2}^{*}} \alpha_{3} e^{-d_{21} \tau_{2}}\left[z_{2}^{2}(t)-z_{2}^{2}\left(t-\tau_{2}\right)\right] \\
& +\frac{\alpha_{2} e^{-d_{11} \tau_{1}}}{2}\left[x^{2}(t)-x^{2}\left(t-\tau_{1}\right)\right]+\frac{\alpha_{3} e^{-d_{21} \tau_{2}}}{2}\left[y_{2}^{2}(t)-y_{2}^{2}\left(t-\tau_{2}\right)\right] \\
= & -\frac{\alpha_{1}}{k} x^{2}(t)-c_{1} x(t) y_{2}(t) \\
& +\alpha_{2} e^{-d_{11} \tau_{1}} x\left(t-\tau_{1}\right) y_{2}(t)+\frac{x^{*}}{y_{2}^{*}} \alpha_{2} e^{-d_{11} \tau_{1}} y_{2}\left(t-\tau_{1}\right) y_{2}(t)-\beta_{1} y_{2}^{2}(t) \\
& -c_{2} y_{2}(t) z_{2}(t)-\frac{x^{*}}{y_{2}^{*}} \alpha_{2} e^{-d_{11} \tau_{1}} y_{2}^{2}(t)-\frac{y_{2}^{*}}{z_{2}^{*}} \alpha_{3} e^{-d_{21} \tau_{2}} z_{2}^{2}(t) \\
& +\alpha_{3} e^{-d_{21} \tau_{2}} y_{2}\left(t-\tau_{2}\right) z_{2}(t)+\frac{y_{2}^{*}}{z_{2}^{*}} \alpha_{3} e^{-d_{21} \tau_{2}} z_{2}\left(t-\tau_{2}\right) z_{2}(t)-\beta_{2} z_{2}^{2}(t)
\end{aligned}
$$




$$
\begin{aligned}
& +\frac{x^{*}}{2 y_{2}^{*}} \alpha_{2} e^{-d_{11} \tau_{1}}\left[y_{2}^{2}(t)-y_{2}^{2}\left(t-\tau_{1}\right)\right] \\
& +\frac{y_{2}^{*}}{2 z_{2}^{*}} \alpha_{3} e^{-d_{21} \tau_{2}}\left[z_{2}^{2}(t)-z_{2}^{2}\left(t-\tau_{2}\right)\right] \\
& +\frac{\alpha_{2} e^{-d_{11} \tau_{1}}}{2}\left[x^{2}(t)-x^{2}\left(t-\tau_{1}\right)\right]+\frac{\alpha_{3} e^{-d_{21} \tau_{2}}}{2}\left[y_{2}^{2}(t)-y_{2}^{2}\left(t-\tau_{2}\right)\right] .
\end{aligned}
$$

Applying fundamental inequality, one has

$$
\begin{aligned}
& \dot{V}\left(x_{t}, y_{1 t}, y_{2 t}, z_{1 t}, z_{2 t}\right) \leq-\frac{\alpha_{1}}{k} x^{2}(t)-c_{1} x(t) y_{2}(t) \\
& +\frac{\alpha_{2} e^{-d_{11} \tau_{1}}}{2}\left[x^{2}\left(t-\tau_{1}\right)+y_{2}^{2}(t)\right] \\
& +\frac{x^{*}}{2 y_{2}^{*}} \alpha_{2} e^{-d_{11} \tau_{1}}\left[y_{2}^{2}\left(t-\tau_{1}\right)+y_{2}^{2}(t)\right] \\
& -\beta_{1} y_{2}^{2}(t)-c_{2} y_{2}(t) z_{2}(t)-\frac{x^{*}}{y_{2}^{*}} \alpha_{2} e^{-d_{11} \tau_{1}} y_{2}^{2}(t) \\
& +\frac{\alpha_{3} e^{-d_{21} \tau_{2}}}{2}\left[y_{2}^{2}\left(t-\tau_{2}\right)+z_{2}^{2}(t)\right] \\
& +\frac{y_{2}^{*}}{2 z_{2}^{*}} \alpha_{3} e^{-d_{21} \tau_{2}}\left[z_{2}^{2}\left(t-\tau_{2}\right)+z_{2}^{2}(t)\right] \\
& -\beta_{2} z_{2}^{2}(t)-\frac{y_{2}^{*}}{z_{2}^{*}} \alpha_{3} e^{-d_{21} \tau_{2}} z_{2}^{2}(t) \\
& +\frac{x^{*}}{2 y_{2}^{*}} \alpha_{2} e^{-d_{11} \tau_{1}}\left[y_{2}^{2}(t)-y_{2}^{2}\left(t-\tau_{1}\right)\right] \\
& +\frac{y_{2}^{*}}{2 z_{2}^{*}} \alpha_{3} e^{-d_{21} \tau_{2}}\left[z_{2}^{2}(t)-z_{2}^{2}\left(t-\tau_{2}\right)\right] \\
& +\frac{\alpha_{2} e^{-d_{11} \tau_{1}}}{2}\left[x^{2}(t)-x^{2}\left(t-\tau_{1}\right)\right] \\
& +\frac{\alpha_{3} e^{-d_{21} \tau_{2}}}{2}\left[y_{2}^{2}(t)-y_{2}^{2}\left(t-\tau_{2}\right)\right] \\
& =-\left(\frac{\alpha_{1}}{k}-\frac{\alpha_{2} e^{-d_{11} \tau_{1}}}{2}\right) x^{2}(t)-c_{1} x(t) y_{2}(t) \\
& -\left(\beta_{1}-\frac{\alpha_{2} e^{-d_{11} \tau_{1}}}{2}-\frac{\alpha_{3} e^{-d_{21} \tau_{2}}}{2}\right) y_{2}^{2}(t)-c_{2} y_{2}(t) z_{2}(t) \\
& -\left(\beta_{2}-\frac{\alpha_{3} e^{-d_{21} \tau_{2}}}{2}\right) z_{2}^{2}(t) .
\end{aligned}
$$

If $2 \alpha_{1}>\alpha_{2} k e^{-d_{11} \tau_{1}}, 2 \beta_{1}>\alpha_{2} e^{-d_{11} \tau_{1}}+\alpha_{3} e^{-d_{21} \tau_{2}}$ and $2 \beta_{2}>\alpha_{3} e^{-d_{21} \tau_{2}}$, then $\dot{V}(t) \leq 0$. With the help of Lyapunov-LaSalle's principle, the equilibrium $(0,0,0,0,0)$ of linearized system (14) is asymptotically stable. Therefore, the interior equilibrium $E^{*}$ of system (2) is stable. This completes the proof.

Remark 3 Incorporating two delays in system (2), the dynamical behaviors are more complicated than the system with one delay (for example, see [8, 10, 14, 24]). Obviously, the method applied in the mentioned papers cannot be applied to system (2) directly. For 
example, when deal with the distribution of characteristic roots for the transcendental equation like $\lambda^{3}+c \lambda^{2}+a_{1} \lambda+a_{2}+\left(b_{1} \lambda+b_{2}\right) e^{-\lambda \tau_{1}}=0$, the local stability of the interior equilibrium $E^{*}$ cannot be derived by Lemma 3.1 [8]. As for this problem, we investigate the stability of the interior equilibrium $E^{*}$ by constructing a suitable Lyapunov functional and applying Lyapunov-LaSalle's principle. That is novel and different from $[8,10,14,24]$.

\section{Asymptotical stability analysis of equilibria}

In the previous section we have found that the trivial equilibrium $E_{0}$ is unstable. In this section, we will discuss the global asymptotic stability for the equilibria $E^{*}, E_{2}$ and $E_{1}$, respectively. For this purpose, we first introduce the following lemma.

Lemma 2 ([35]) Consider the following equation:

$$
\dot{\vartheta}(t)=\varrho \vartheta(t-\tau)-\varsigma \vartheta(t)-\varpi \vartheta^{2}(t)
$$

where all parameters are positive constants, $\vartheta(t)>0$ for $t \in[-\tau, 0]$, one has

(i) If $\varrho>\varsigma$, then $\lim _{t \rightarrow+\infty} \vartheta(t)=\frac{\varrho-\varsigma}{\varpi}$.

(ii) If $\varrho<\varsigma$, then $\lim _{t \rightarrow+\infty} \vartheta(t)=0$.

By Lemma 2 and using an iterative technique, we can obtain the following theorems.

Theorem 7 Under the conditions (C2), (C3) and (C4), further suppose that

$$
\begin{aligned}
& 2 \alpha_{1}>\alpha_{2} k e^{-d_{11} \tau_{1}}, \\
& 2 \beta_{1}>\alpha_{2} e^{-d_{11} \tau_{1}}+\alpha_{3} e^{-d_{21} \tau_{2}}, \\
& 2 \beta_{2}>\alpha_{3} e^{-d_{21} \tau_{2}}
\end{aligned}
$$

and

$$
\alpha_{1} \beta_{1} \beta_{2}>\alpha_{1} \alpha_{3} c_{2} e^{-d_{21} \tau_{2}}+\alpha_{2} \beta_{2} c_{1} k e^{-d_{11} \tau_{1}},
$$

then the interior equilibrium $E^{*}$ of system (2) is AS.

Proof Under the conditions (C2), (C3) and (C4), if $2 \alpha_{1}>\alpha_{2} k e^{-d_{11} \tau_{1}}, 2 \beta_{1}>\alpha_{2} e^{-d_{11} \tau_{1}}+$ $\alpha_{3} e^{-d_{21} \tau_{2}}$ and $2 \beta_{2}>\alpha_{3} e^{-d_{21} \tau_{2}}$, by Theorem 6 , one find that the interior equilibrium $E^{*}$ is stable. Therefore, we need only prove that $\lim _{t \rightarrow+\infty}\left(x(t), y_{1}(t), y_{2}(t), z_{1}(t), z_{2}(t)\right)=$ $\left(x^{*}, y_{1}^{*}, y_{2}^{*}, z_{1}^{*}, z_{2}^{*}\right)$.

Define

$$
\begin{array}{ll}
U_{1}=\limsup _{t \rightarrow+\infty} x(t), & V_{1}=\liminf _{t \rightarrow+\infty} x(t), \\
U_{2}=\limsup _{t \rightarrow+\infty} y_{2}(t), & V_{2}=\liminf _{t \rightarrow+\infty} y_{2}(t), \\
U_{3}=\limsup _{t \rightarrow+\infty} z_{2}(t), & V_{3}=\liminf _{t \rightarrow+\infty} z_{2}(t),
\end{array}
$$

in the next, we will state and prove that $U_{1}=V_{1}=x^{*}, U_{2}=V_{2}=y_{2}^{*}, U_{3}=V_{3}=z_{2}^{*}$. 
From the first equation of system (2), we obtain

$$
\dot{x}(t) \leq x(t)\left(\alpha_{1}\left(1-\frac{x(t)}{k}\right)\right) .
$$

By the comparison theorem, one has

$$
U_{1}=\limsup _{t \rightarrow+\infty} x(t) \leq k \stackrel{\text { def }}{=} N_{1}^{x}
$$

Since $\varepsilon>0$ is sufficiently small, then there exists a $T_{11}>0$ such that $x(t) \leq N_{1}^{x}+\varepsilon$ for $t>T_{11}$. We obtain from the third equation of system (2), for $t>T_{11}+\tau$,

$$
\dot{y}_{2}(t) \leq \alpha_{2} e^{-d_{11} \tau_{1}}\left(N_{1}^{x}+\varepsilon\right) y_{2}\left(t-\tau_{1}\right)-d_{12} y_{2}(t)-\beta_{1} y_{2}^{2}(t) .
$$

By constructing the following auxiliary equation:

$$
\dot{\mathfrak{v}}(t)=\alpha_{2} e^{-d_{11} \tau_{1}}\left(N_{1}^{x}+\varepsilon\right) \mathfrak{v}\left(t-\tau_{1}\right)-d_{12} \mathfrak{v}(t)-\beta_{1} \mathfrak{v}^{2}(t) .
$$

Noting that condition (C4) implies that $\alpha_{2} k e^{-d_{11} \tau_{1}}>d_{12}$, and so, by applying Lemma 2(i), we obtain that

$$
\lim _{t \rightarrow+\infty} \mathfrak{v}(t)=\frac{\alpha_{2} e^{-d_{11} \tau_{1}}\left(N_{1}^{x}+\varepsilon\right)-d_{12}}{\beta_{1}} .
$$

Using the comparison theorem,

$$
U_{2}=\limsup _{t \rightarrow+\infty} y_{2}(t) \leq \frac{\alpha_{2} e^{-d_{11} \tau_{1}}\left(N_{1}^{x}+\varepsilon\right)-d_{12}}{\beta_{1}}
$$

Let $N_{1}^{y}=\frac{\alpha_{2} e^{-d_{11} \tau_{1}} N_{1}^{x}-d_{12}}{\beta_{1}}$, since $\varepsilon>0$ sufficiently small, thereby, $U_{2} \leq N_{1}^{y}$. Consequently, there exists a $T_{12} \geq T_{11}+\tau$ such that $y_{2}(t) \leq N_{1}^{y}+\varepsilon$ for $t>T_{12}$.

From the fifth equation of system (2), we have

$$
\dot{z}_{2}(t) \leq \alpha_{3} e^{-d_{21} \tau_{2}}\left(N_{1}^{y}+\varepsilon\right) z_{2}\left(t-\tau_{2}\right)-d_{22} z_{2}(t)-\beta_{2} z_{2}^{2}(t) \quad \text { for } t>T_{12}+\tau \text {. }
$$

Using Lemma 2(i) and comparison theorem, one can get

$$
U_{3}=\limsup _{t \rightarrow+\infty} z_{2}(t) \leq \frac{\alpha_{3} e^{-d_{21} \tau_{2}}\left(N_{1}^{y}+\varepsilon\right)-d_{22}}{\beta_{2}} .
$$

Let $N_{1}^{z}=\frac{\alpha_{3} e^{-d_{21} \tau_{2}} N_{1}^{y}-d_{22}}{\beta_{2}}$, since $\varepsilon>0$ sufficiently small, so we obtain $U_{3} \leq N_{1}^{z}$. Therefore, there exists a $T_{21} \geq T_{12}+\tau$ such that $z_{2}(t) \leq N_{1}^{z}+\varepsilon$ for $t>T_{21}$.

We obtain from the first equation of system (2), for $t>T_{12}+\tau$,

$$
\dot{x}(t) \geq x(t)\left[\alpha_{1}\left(1-\frac{x(t)}{k}\right)-c_{1}\left(N_{1}^{y}+\varepsilon\right)\right] .
$$


Using the comparison theorem,

$$
V_{1}=\liminf _{t \rightarrow+\infty} x(t) \geq \frac{k\left[\alpha_{1}-c_{1}\left(N_{1}^{y}+\varepsilon\right)\right]}{\alpha_{1}} .
$$

Let $M_{1}^{x}=\frac{k\left(\alpha_{1}-c_{1} N_{1}^{y}\right)}{\alpha_{1}}$, since $\varepsilon>0$ is sufficiently small, then one has $V_{1} \geq M_{1}^{x}$. Hence, there is a $T_{22} \geq T_{12}+\tau$ such that $x(t) \geq M_{1}^{x}-\varepsilon$ for $t>T_{22}$.

From the third equation of system (2) we obtain, for $t>\max \left\{T_{21}, T_{22}\right\}$,

$$
\dot{y}_{2}(t) \geq \alpha_{2} e^{-d_{11} \tau_{1}}\left(M_{1}^{x}-\varepsilon\right) y_{2}\left(t-\tau_{1}\right)-d_{12} y_{2}(t)-\beta_{1} y_{2}^{2}(t)-c_{2}\left(N_{1}^{z}+\varepsilon\right) y_{2}(t) .
$$

By applying Lemma 2(i) and the standard comparison theorem, then

$$
V_{2}=\liminf _{t \rightarrow+\infty} y_{2}(t) \geq \frac{\alpha_{2} e^{-d_{11} \tau_{1}}\left(M_{1}^{x}-\varepsilon\right)-d_{12}-c_{2}\left(N_{1}^{z}+\varepsilon\right)}{\beta_{1}} .
$$

Let $M_{1}^{y}=\frac{\alpha_{2} e^{-d_{11} \tau_{1}} M_{1}^{x}-d_{12}-c_{2} N_{1}^{z}}{\beta_{1}}$, since $\varepsilon>0$ sufficiently small, obviously, $V_{2} \geq M_{1}^{y}$. Consequently, there exists a $T_{31} \geq \max \left\{T_{21}, T_{22}\right\}$ such that $y_{2}(t) \geq M_{1}^{y}-\varepsilon$ for $t>T_{31}$.

We obtain from the fifth equation of system (2), for $t>T_{31}+\tau$,

$$
\dot{z}_{2}(t) \geq \alpha_{3} e^{-d_{21} \tau_{2}}\left(M_{1}^{y}-\varepsilon\right) z_{2}\left(t-\tau_{2}\right)-d_{22} z_{2}(t)-\beta_{2} z_{2}^{2}(t) .
$$

From this differential inequality, by applying Lemma 2(i), one can get

$$
V_{3}=\liminf _{t \rightarrow+\infty} z_{2}(t) \geq \frac{\alpha_{3} e^{-d_{21} \tau_{2}}\left(M_{1}^{y}-\varepsilon\right)-d_{22}}{\beta_{2}} .
$$

Let $M_{1}^{z}=\frac{\alpha_{3} e^{-d_{21} \tau_{2}} M_{1}^{y}-d_{22}}{\beta_{2}}$, since $\varepsilon>0$ is sufficiently small, then $V_{3} \geq M_{1}^{z}$. Hence, there exists a $T_{32} \geq T_{31}+\tau$ such that $z_{2}(t) \geq M_{1}^{z}-\varepsilon$ for $t>T_{32}$.

Similar to the above discussion, we obtain from the first equation of system (2), for $t>$ $T_{31}$,

$$
\dot{x}(t) \leq x(t)\left[\alpha_{1}\left(1-\frac{x(t)}{k}\right)-c_{1}\left(M_{1}^{y}-\varepsilon\right)\right] .
$$

By comparison,

$$
U_{1}=\limsup _{t \rightarrow+\infty} x(t) \leq \frac{k\left[\alpha_{1}-c_{1}\left(M_{1}^{y}-\varepsilon\right)\right]}{\alpha_{1}} .
$$

Let $N_{2}^{x}=\frac{k\left(\alpha_{1}-c_{1} M_{1}^{y}\right)}{\alpha_{1}}$, since $\varepsilon>0$ is sufficiently small, thereby, $U_{1} \leq N_{2}^{x}$. Thus, there exists a $T_{33} \geq T_{31}+\tau$ such that $x(t) \leq N_{2}^{x}+\varepsilon$ for $t>T_{33}$.

We obtain from the third equation of system (2), for $t>\max \left\{T_{32}, T_{33}\right\}$,

$$
\dot{y}_{2}(t) \leq \alpha_{2} e^{-d_{11} \tau_{1}}\left(N_{2}^{x}+\varepsilon\right) y_{2}\left(t-\tau_{1}\right)-d_{12} y_{2}(t)-\beta_{1} y_{2}^{2}(t)-c_{2}\left(M_{1}^{z}-\varepsilon\right) y_{2}(t) .
$$

By applying Lemma 2(i) and comparison, we obtain that

$$
U_{2}=\limsup _{t \rightarrow+\infty} y_{2}(t) \leq \frac{\alpha_{2} e^{-d_{11} \tau_{1}}\left(N_{2}^{x}+\varepsilon\right)-d_{12}-c_{2}\left(M_{1}^{z}-\varepsilon\right)}{\beta_{1}} .
$$


Let $N_{2}^{y}=\frac{\alpha_{2} e^{-d_{11} \tau_{1}} N_{2}^{x}-d_{12}-c_{2} M_{1}^{z}}{\beta_{1}}$, since $\varepsilon>0$ is sufficiently small, so one has $U_{2} \leq N_{2}^{y}$ holds. Therefore, there exists a $T_{41} \geq \max \left\{T_{32}, T_{33}\right\}$ such that $y_{2}(t) \leq N_{2}^{y}+\varepsilon$ for $t>T_{41}$.

From the fifth equation of system (2), for $t>T_{41}$,

$$
\dot{z}_{2}(t) \leq \alpha_{3} e^{-d_{21} \tau_{2}}\left(N_{2}^{y}+\varepsilon\right) z_{2}\left(t-\tau_{2}\right)-d_{22} z_{2}(t)-\beta_{2} z_{2}^{2}(t) .
$$

Similarly, we get

$$
U_{3}=\limsup _{t \rightarrow+\infty} z_{2}(t) \leq \frac{\alpha_{3} e^{-d_{21} \tau_{2}}\left(N_{2}^{y}+\varepsilon\right)-d_{22}}{\beta_{2}} .
$$

Let $N_{2}^{z}=\frac{\alpha_{3} e^{-d_{21} \tau_{2} N_{2}^{y}-d_{22}}}{\beta_{2}}$, since $\varepsilon>0$ is sufficiently small, then we find that $U_{3} \leq N_{2}^{z}$ holds. Consequently, there exists a $T_{42} \geq T_{41}$ such that $z_{2}(t) \leq N_{2}^{z}+\varepsilon$ for $t>T_{42}$.

We obtain from the first equation of system (2)

$$
\dot{x}(t) \geq x(t)\left[\alpha_{1}\left(1-\frac{x(t)}{k}\right)-c_{1}\left(N_{2}^{y}+\varepsilon\right)\right] \text { for } t>T_{41}+\tau .
$$

Using a comparison argument,

$$
V_{1}=\liminf _{t \rightarrow+\infty} x(t) \geq \frac{k\left[\alpha_{1}-c_{1}\left(N_{2}^{y}+\varepsilon\right)\right]}{\alpha_{1}} .
$$

Let $M_{2}^{x}=\frac{k\left(\alpha_{1}-c_{1} N_{2}^{y}\right)}{\alpha_{1}}$, since $\varepsilon>0$ is sufficiently small, then obviously $V_{1} \geq M_{2}^{x}$ holds. Hence, there is a $T_{43} \geq T_{41}+\tau$ such that $x(t) \geq M_{2}^{x}-\varepsilon$ for $t>T_{43}$.

We obtain from the third equation of system (2), for $t>\max \left\{T_{42}, T_{43}\right\}$,

$$
\dot{y}_{2}(t) \geq \alpha_{2} e^{-d_{11} \tau_{1}}\left(M_{2}^{x}-\varepsilon\right) y_{2}\left(t-\tau_{1}\right)-d_{12} y_{2}(t)-\beta_{1} y_{2}^{2}(t)-c_{2}\left(N_{2}^{z}+\varepsilon\right) y_{2}(t) .
$$

By applying Lemma 2(i), one can get

$$
V_{2}=\liminf _{t \rightarrow+\infty} y_{2}(t) \geq \frac{\alpha_{2} e^{-d_{11} \tau_{1}}\left(M_{2}^{x}-\varepsilon\right)-d_{12}-c_{2}\left(N_{2}^{z}+\varepsilon\right)}{\beta_{1}} .
$$

Let $M_{2}^{y}=\frac{\alpha_{2} e^{-d_{11} \tau_{1}} M_{2}^{x}-d_{12}-c_{2} N_{2}^{z}}{\beta_{1}}$, since $\varepsilon>0$ is sufficiently small, then one has $V_{2} \geq M_{2}^{y}$ holds. Thus, there exists a $T_{51} \geq \max \left\{T_{42}, T_{43}\right\}+\tau$ such that $y_{2}(t) \geq M_{2}^{y}-\varepsilon$ for $t>T_{51}$.

From the fifth equation of system (2), for $t>T_{51}$,

$$
\dot{z}_{2}(t) \geq \alpha_{3} e^{-d_{21} \tau_{2}}\left(M_{2}^{y}-\varepsilon\right) z_{2}\left(t-\tau_{2}\right)-d_{22} z_{2}(t)-\beta_{2} z_{2}^{2}(t) .
$$

By applying Lemma 2(i) and the standard comparison theorem, one obtains

$$
V_{3}=\liminf _{t \rightarrow+\infty} z_{2}(t) \geq \frac{\alpha_{3} e^{-d_{21} \tau_{2}}\left(M_{2}^{y}-\varepsilon\right)-d_{22}}{\beta_{2}} .
$$

Let $M_{2}^{z}=\frac{\alpha_{3} e^{-d_{21} \tau_{2}} M_{2}^{y}-d_{22}}{\beta_{2}}$, since $\varepsilon>0$ is sufficiently small, thereby, $V_{3} \geq M_{2}^{z}$ holds. Therefore, there exists a $T_{52} \geq T_{51}$ such that $z_{2}(t) \geq M_{2}^{z}-\varepsilon$ for $t>T_{52}$. 
So far, we have completed the first step of the iterative scheme. Repeating the above argument and using mathematical induction, we obtain six sequences $N_{n}^{x}, N_{n}^{y}, N_{n}^{z}, M_{n}^{x}$, $M_{n}^{y}, M_{n}^{z}, n=1,2, \ldots$, such that, for $n \geq 2$,

$$
\begin{aligned}
& N_{n}^{x}=\frac{k\left(\alpha_{1}-c_{1} M_{n-1}^{y}\right)}{\alpha_{1}}, \quad N_{n}^{y}=\frac{\alpha_{2} e^{-d_{11} \tau_{1}} N_{n}^{x}-d_{12}-c_{2} M_{n-1}^{z}}{\beta_{1}}, \\
& M_{n}^{x}=\frac{k\left(\alpha_{1}-c_{1} N_{n}^{y}\right)}{\alpha_{1}}, \quad M_{n}^{y}=\frac{\alpha_{2} e^{-d_{11} \tau_{1}} M_{n}^{x}-d_{12}-c_{2} N_{n}^{z}}{\beta_{1}}, \\
& N_{n}^{z}=\frac{\alpha_{3} e^{-d_{21} \tau_{2}} N_{n}^{y}-d_{22}}{\beta_{2}}, \quad M_{n}^{z}=\frac{\alpha_{3} e^{-d_{21} \tau_{2}} M_{n}^{y}-d_{22}}{\beta_{2}} .
\end{aligned}
$$

By analyzing, one can get

$$
M_{n}^{x} \leq V_{1} \leq U_{1} \leq N_{n}^{x}, \quad M_{n}^{y} \leq V_{2} \leq U_{2} \leq N_{n}^{y}, \quad M_{n}^{z} \leq V_{3} \leq U_{3} \leq N_{n}^{z} .
$$

From (17), we obtain that

$$
N_{n+1}^{y}=\beta_{1}^{-1}\left(1-\beta_{1}^{-1} \Phi\right)\left(\Phi+\beta_{1}\right) y_{2}^{*}+\left(\beta_{1}^{-1} \Phi\right)^{2} N_{n}^{y},
$$

where

$$
\Phi=\alpha_{1}^{-1} \alpha_{2} c_{1} k e^{-d_{11} \tau_{1}}+\alpha_{3} \beta_{2}^{-1} c_{2} e^{-d_{21} \tau_{2}} .
$$

As $N_{n}^{y} \geq y_{2}^{*}$ and $\alpha_{1} \beta_{1} \beta_{2}>\alpha_{1} \alpha_{3} c_{2} e^{-d_{21} \tau_{2}}+\alpha_{2} \beta_{2} c_{1} k e^{-d_{11} \tau_{1}}$, one can obtain from (19) that

$$
\begin{aligned}
N_{n+1}^{y}-N_{n}^{y} & =\beta_{1}^{-1}\left(1-\beta_{1}^{-1} \Phi\right)\left(\Phi+\beta_{1}\right) y_{2}^{*}+\left[\left(\beta_{1}^{-1} \Phi\right)^{2}-1\right] N_{n}^{y} \\
& \leq\left(1-\beta_{1}^{-1} \Phi\right)\left(1+\beta_{1}^{-1} \Phi\right) y_{2}^{*}+\left[\left(\beta_{1}^{-1} \Phi\right)^{2}-1\right] y_{2}^{*} \\
& =0 .
\end{aligned}
$$

Consequently, the sequence $N_{n}^{y}$ is monotonically decreasing and

$$
\lim _{n \rightarrow+\infty} N_{n}^{y}=\frac{\alpha_{1}\left(\alpha_{2} \beta_{2} k e^{-d_{11} \tau_{1}}-\beta_{2} d_{12}+c_{2} d_{22}\right)}{\alpha_{1} \beta_{1} \beta_{2}+\alpha_{1} \alpha_{3} c_{2} e^{-d_{21} \tau_{2}}+\alpha_{2} \beta_{2} c_{1} k e^{-d_{11} \tau_{1}}}=y_{2}^{*} .
$$

Therefore, from (17) and (20) we see that the sequences $N_{n}^{x}$ and $N_{n}^{z}$ are decreasing and the sequences $M_{n}^{x}, M_{n}^{y}$ and $M_{n}^{z}$ are increasing, furthermore,

$$
\begin{array}{ll}
\lim _{n \rightarrow+\infty} N_{n}^{x}=x^{*}, & \lim _{n \rightarrow+\infty} N_{n}^{z}=z_{2}^{*}, \\
\lim _{n \rightarrow+\infty} M_{n}^{x}=x^{*}, & \lim _{n \rightarrow+\infty} M_{n}^{y}=y_{2}^{*},
\end{array}
$$

Hence,

$$
\lim _{t \rightarrow+\infty} x(t)=x^{*}, \quad \lim _{t \rightarrow+\infty} y_{2}(t)=y_{2}^{*}, \quad \lim _{t \rightarrow+\infty} z_{2}(t)=z_{2}^{*} .
$$

We obtain from (6)

$$
y_{1}(t)=\frac{\alpha_{2} \int_{t-\tau_{1}}^{t} e^{d_{11} s} x(s) y_{2}(s) d s}{e^{d_{11} t}} .
$$


According to L'Hospital's rule, one can get

$$
\begin{aligned}
\lim _{t \rightarrow+\infty} y_{1}(t) & =\lim _{t \rightarrow+\infty} \frac{\alpha_{2}\left[e^{d_{11} t} x(t) y_{2}(t)-e^{d_{11}\left(t-\tau_{1}\right)} x\left(t-\tau_{1}\right) y_{2}\left(t-\tau_{1}\right)\right]}{d_{11} e^{d_{11} t}} \\
& =\frac{\alpha_{2}}{d_{11}} \lim _{t \rightarrow+\infty}\left[x(t) y_{2}(t)-e^{-d_{11} \tau_{1}} x\left(t-\tau_{1}\right) y_{2}\left(t-\tau_{1}\right)\right] \\
& =\frac{\alpha_{2}}{d_{11}}\left(1-e^{-d_{11} \tau_{1}}\right) x^{*} y_{2}^{*}=y_{1}^{*} .
\end{aligned}
$$

We obtain from (7) that

$$
z_{1}(t)=\frac{\alpha_{3} \int_{t-\tau_{2}}^{t} e^{d_{21} s} z_{2}(s) y_{2}(s) d s}{e^{d_{21} t}} .
$$

According to L'Hospital's rule, one has

$$
\begin{aligned}
\lim _{t \rightarrow+\infty} z_{1}(t) & =\lim _{t \rightarrow+\infty} \frac{\alpha_{3}\left[e^{d_{21} t} y_{2}(t) z_{2}(t)-e^{d_{21}\left(t-\tau_{2}\right)} y_{2}\left(t-\tau_{2}\right) z_{2}\left(t-\tau_{2}\right)\right]}{d_{21} e^{d_{21} t}} \\
& =\frac{\alpha_{3}}{d_{21}} \lim _{t \rightarrow+\infty}\left[y_{2}(t) z_{2}(t)-e^{-d_{21} \tau_{2}} y_{2}\left(t-\tau_{1}\right) z_{2}\left(t-\tau_{1}\right)\right] \\
& =\frac{\alpha_{3}}{d_{21}}\left(1-e^{-d_{21} \tau_{2}}\right) y_{2}^{*} z_{2}^{*}=z_{1}^{*} .
\end{aligned}
$$

This completes the proof.

In the next, we will discuss the global stability of the boundary equilibrium $E_{2}\left(x^{0}, y_{1}^{0}, y_{2}^{0}, 0,0\right)$ of system (2) when

$$
\alpha_{1} \alpha_{3} e^{-d_{21} \tau_{2}}\left(\alpha_{2} k e^{-d_{11} \tau_{1}}-d_{12}\right)-d_{22}\left(\alpha_{1} \beta_{1}+\alpha_{2} c_{1} k e^{-d_{11} \tau_{1}}\right)<0 .
$$

Theorem 8 The delays have great impacts on the dynamics for system (2). More precisely, let $m_{1}=\frac{1}{d_{11}} \ln \frac{\alpha_{2} c_{1} k}{\alpha_{1} \beta_{1}}, m_{2}=\frac{1}{d_{11}} \ln \frac{\alpha_{2} k}{d_{12}}$ and $m_{4}=\max \left\{\frac{1}{d_{21}} \ln \frac{\alpha_{3}\left(\alpha_{1} \beta_{1}-c_{1} d_{12}\right)}{\beta_{1} c_{1} d_{22}}, \frac{1}{d_{21}} \ln \frac{\alpha_{3} y_{2}^{0}}{d_{22}}\right\}$, if $\tau_{1} \in$ $\left(m_{1}, m_{2}\right)$ and $\tau_{2} \in\left(m_{4},+\infty\right)$, then the boundary equilibrium $E_{2}$ of system (2) is AS.

Proof By $\tau_{1} \in\left(m_{1}, m_{2}\right)$, one finds that (C1) and $\alpha_{1} \beta_{1}-\alpha_{2} c_{1} k e^{-d_{11} \tau_{1}}>0$ hold. Thus, the boundary equilibrium $E_{2}$ exists. At the same time, by $\tau_{2} \in\left(m_{4},+\infty\right)$, it is obvious that $\alpha_{3} e^{-d_{21} \tau_{2}}\left(\alpha_{2} k e^{-d_{11} \tau_{1}}-d_{12}\right)-\beta_{1} d_{22}<0$ and $\alpha_{3} y_{2}^{0} e^{-d_{21} \tau_{2}}<d_{22}$.

Using Theorem 5 , we have found that the boundary equilibrium $E_{2}\left(x^{0}, y_{1}^{0}, y_{2}^{0}, 0,0\right)$ is LAS. Therefore, it is sufficient to show that $\lim _{t \rightarrow+\infty}\left(x(t), y_{1}(t), y_{2}(t), z_{1}(t), z_{2}(t)\right)=\left(x^{0}, y_{1}^{0}, y_{2}^{0}, 0,0\right)$.

Since $\alpha_{2} e^{-d_{11} \tau_{1}}(k+\varepsilon)>d_{12}$, the same arguments as those in the proof of Theorem 7 show that (15), (16) hold, i.e.,

$$
\begin{aligned}
& U_{1}=\limsup _{t \rightarrow+\infty} x(t) \leq k \stackrel{\text { def }}{=} N_{1}^{x}, \\
& U_{2}=\limsup _{t \rightarrow+\infty} y_{2}(t) \leq \frac{\alpha_{2} e^{-d_{11} \tau_{1}} N_{1}^{x}-d_{12}}{\beta_{1}} \stackrel{\text { def }}{=} N_{1}^{y} .
\end{aligned}
$$

Hence, for $\varepsilon>0$ sufficiently small, there is a $T_{12} \geq T_{11}+\tau$ such that $y_{2}(t) \leq N_{1}^{y}+\varepsilon$ for $t>T_{12}$. 
We obtain from the fifth equation of system (2), for $t>T_{12}+\tau$,

$$
\dot{z}_{2}(t) \leq \alpha_{3} e^{-d_{21} \tau_{2}}\left(N_{1}^{y}+\varepsilon\right) z_{2}\left(t-\tau_{2}\right)-d_{22} z_{2}(t)-\beta_{2} z_{2}^{2}(t)
$$

By applying Lemma 2(ii) and the standard comparison theorem, one has

$$
\lim _{t \rightarrow+\infty} z_{2}(t)=0
$$

Thus, for $\varepsilon>0$ sufficiently small, there exists a $T_{21} \geq T_{12}+\tau$ such that $0<z_{2}(t)<\varepsilon$ for $t>T_{21}$.

We obtain from the first equation of system (2), for $t>T_{12}+\tau$,

$$
\dot{x}(t) \geq x(t)\left[\alpha_{1}\left(1-\frac{x(t)}{k}\right)-c_{1}\left(N_{1}^{y}+\varepsilon\right)\right] .
$$

By the comparison theorem,

$$
V_{1}=\liminf _{t \rightarrow+\infty} x(t) \geq \frac{k\left[\alpha_{1}-c_{1}\left(N_{1}^{y}+\varepsilon\right)\right]}{\alpha_{1}} .
$$

Let $M_{1}^{x}=\frac{k\left(\alpha_{1}-c_{1} N_{1}^{y}\right)}{\alpha_{1}}$, since $\varepsilon>0$ is sufficiently small, obviously, $V_{1} \geq M_{1}^{x}$ holds. Therefore, there exists a $T_{22} \geq T_{12}+\tau$ such that $x(t) \geq M_{1}^{x}-\varepsilon$ for $t>T_{22}$.

We obtain from the third equation of system (2), for $t>\max \left\{T_{21}, T_{22}\right\}$,

$$
\dot{y}_{2}(t) \geq \alpha_{2} e^{-d_{11} \tau_{1}}\left(M_{1}^{x}-\varepsilon\right) y_{2}\left(t-\tau_{1}\right)-d_{12} y_{2}(t)-\beta_{1} y_{2}^{2}(t)-c_{2} \varepsilon y_{2}(t) .
$$

By applying Lemma 2(i) and the standard comparison theorem, one has

$$
V_{2}=\liminf _{t \rightarrow+\infty} y_{2}(t) \geq \frac{\alpha_{2} e^{-d_{11} \tau_{1}}\left(M_{1}^{x}-\varepsilon\right)-d_{12}-c_{2} \varepsilon}{\beta_{1}} .
$$

Let $M_{1}^{y}=\frac{\alpha_{2} e^{-d_{11} \tau_{1}} M_{1}^{x}-d_{12}}{\beta_{1}}$, since $\varepsilon>0$ sufficiently small, so we get $V_{2} \geq M_{1}^{y}$. Consequently, there exists a $T_{31} \geq \max \left\{T_{21}, T_{22}\right\}$ such that $y_{2}(t) \geq M_{1}^{y}-\varepsilon$ for $t>T_{31}$.

Similar to the above discussion, we obtain from the first equation of system (2), for $t>$ $T_{31}+\tau$,

$$
\dot{x}(t) \leq x(t)\left[\alpha_{1}\left(1-\frac{x(t)}{k}\right)-c_{1}\left(M_{1}^{y}-\varepsilon\right)\right] .
$$

By the comparison theorem,

$$
U_{1}=\limsup _{t \rightarrow+\infty} x(t) \leq \frac{k\left[\alpha_{1}-c_{1}\left(M_{1}^{y}-\varepsilon\right)\right]}{\alpha_{1}} .
$$

Let $N_{2}^{x}=\frac{k\left(\alpha_{1}-c_{1} M_{1}^{y}\right)}{\alpha_{1}}$, for $\varepsilon>0$ sufficiently small, one has $U_{1} \geq N_{2}^{x}$. Hence, there exists a $T_{32} \geq T_{31}+\tau$ such that $x(t) \leq N_{2}^{x}+\varepsilon$ for $t>T_{33}$.

We obtain from the third equation of system (2), for $t>\max \left\{T_{32}, T_{21}\right\}$,

$$
\dot{y}_{2}(t) \leq \alpha_{2} e^{-d_{11} \tau_{1}}\left(N_{2}^{x}+\varepsilon\right) y_{2}\left(t-\tau_{1}\right)-d_{12} y_{2}(t)-\beta_{1} y_{2}^{2}(t) .
$$


By applying Lemma 2(i) and comparison, one can get

$$
U_{2}=\limsup _{t \rightarrow+\infty} y_{2}(t) \leq \frac{\alpha_{2} e^{-d_{11} \tau_{1}}\left(N_{2}^{x}+\varepsilon\right)-d_{12}}{\beta_{1}}
$$

Let $N_{2}^{y}=\frac{\alpha_{2} e^{-d_{11} \tau_{1}} N_{2}^{x}-d_{12}}{\beta_{1}}$, since $\varepsilon>0$ is sufficiently small, thereby, $U_{2} \leq N_{2}^{y}$. Accordingly, there exists a $T_{41} \geq \max \left\{T_{32}, T_{21}\right\}$ such that $y_{2}(t) \leq N_{2}^{y}+\varepsilon$ for $t>T_{41}$.

From the first equation of system (2), for $t>T_{41}+\tau$,

$$
\dot{x}(t) \geq x(t)\left[\alpha_{1}\left(1-\frac{x(t)}{k}\right)-c_{1}\left(N_{2}^{y}+\varepsilon\right)\right] .
$$

By the comparison theorem,

$$
V_{1}=\liminf _{t \rightarrow+\infty} x(t) \geq \frac{k\left[\alpha_{1}-c_{1}\left(N_{2}^{y}+\varepsilon\right)\right]}{\alpha_{1}} .
$$

Let $M_{2}^{x}=\frac{k\left(\alpha_{1}-c_{1} N_{2}^{y}\right)}{\alpha_{1}}$, since $\varepsilon>0$ is sufficiently small, then obviously $V_{1} \geq M_{2}^{x}$. Therefore, there exists a $T_{42} \geq T_{41}+\tau$ such that $x(t) \geq M_{2}^{x}-\varepsilon$ for $t>T_{42}$.

We obtain from the third equation of system (2), for $t>\max \left\{T_{42}, T_{21}\right\}$,

$$
\dot{y}_{2}(t) \geq \alpha_{2} e^{-d_{11} \tau_{1}}\left(M_{2}^{x}-\varepsilon\right) y_{2}\left(t-\tau_{1}\right)-d_{12} y_{2}(t)-\beta_{1} y_{2}^{2}(t)-c_{2} \varepsilon y_{2}(t) .
$$

By applying Lemma 2(i), one has

$$
V_{2}=\liminf _{t \rightarrow+\infty} y_{2}(t) \geq \frac{\alpha_{2} e^{-d_{11} \tau_{1}}\left(M_{2}^{x}-\varepsilon\right)-d_{12}-c_{2} \varepsilon}{\beta_{1}} .
$$

Let $M_{2}^{y}=\frac{\alpha_{2} e^{-d_{11} \tau_{1}} M_{2}^{x}-d_{12}}{\beta_{1}}$, for $\varepsilon>0$ sufficiently small, so we can get $V_{2} \geq M_{2}^{y}$. Consequently, there exists a $T_{51} \geq \max \left\{T_{42}, T_{21}\right\}+\tau$ such that $y_{2}(t) \geq M_{2}^{y}-\varepsilon$ for $t>T_{51}$.

So far, we have completed the first step of the iterative scheme. Repeating the above argument and using mathematical induction, we obtain four sequences $N_{n}^{x}, N_{n}^{y}, M_{n}^{x}, M_{n}^{y}$, $n=1,2, \ldots$, such that, for $n \geq 2$,

$$
\begin{aligned}
& N_{n}^{x}=\frac{k\left(\alpha_{1}-c_{1} M_{n-1}^{y}\right)}{\alpha_{1}}, \quad N_{n}^{y}=\frac{\alpha_{2} e^{-d_{11} \tau_{1}} N_{n}^{x}-d_{12}}{\beta_{1}}, \\
& M_{n}^{x}=\frac{k\left(\alpha_{1}-c_{1} N_{n}^{y}\right)}{\alpha_{1}}, \quad M_{n}^{y}=\frac{\alpha_{2} e^{-d_{11} \tau_{1}} M_{n}^{x}-d_{12}}{\beta_{1}} .
\end{aligned}
$$

By analyzing, we can get

$$
M_{n}^{x} \leq V_{1} \leq U_{1} \leq N_{n}^{x}, \quad M_{n}^{y} \leq V_{2} \leq U_{2} \leq N_{n}^{y} .
$$

From (26), one has

$$
N_{n+1}^{y}=\frac{\left(\alpha_{1} \beta_{1}-\alpha_{2} c_{1} k e^{-d_{11} \tau_{1}}\right)\left(\alpha_{2} k e^{-d_{11} \tau_{1}}-d_{12}\right)}{\alpha_{1} \beta_{1}^{2}}+\left(\frac{\alpha_{2} c_{1} k e^{-d_{11} \tau_{1}}}{\alpha_{1} \beta_{1}}\right)^{2} N_{n}^{y} .
$$


As $N_{n}^{y} \geq y_{2}^{0}$, we can obtain from (28)

$$
\begin{aligned}
N_{n+1}^{y}-N_{n}^{y}= & \frac{\left(\alpha_{1} \beta_{1}-\alpha_{2} c_{1} k e^{-d_{11} \tau_{1}}\right)\left(\alpha_{1} \beta_{1}+\alpha_{2} c_{1} k e^{-d_{11} \tau_{1}}\right)}{\left(\alpha_{1} \beta_{1}^{2}\right)^{2}} y_{2}^{0} \\
& +\left[\left(\frac{\alpha_{2} c_{1} k e^{-d_{11} \tau_{1}}}{\alpha_{1} \beta_{1}}\right)^{2}-1\right] N_{n}^{y} \\
\leq & \frac{\left(\alpha_{1} \beta_{1}-\alpha_{2} c_{1} k e^{-d_{11} \tau_{1}}\right)\left(\alpha_{1} \beta_{1}+\alpha_{2} c_{1} k e^{-d_{11} \tau_{1}}\right)}{\left(\alpha_{1} \beta_{1}^{2}\right)^{2}} y_{2}^{0} \\
& +\frac{\left(\alpha_{2} c_{1} k e^{-d_{11} \tau_{1}}-\alpha_{1} \beta_{1}\right)\left(\alpha_{2} c_{1} k e^{-d_{11} \tau_{1}}+\alpha_{1} \beta_{1}\right)}{\left(\alpha_{1} \beta_{1}^{2}\right)^{2}} y_{2}^{0} \\
= & 0 .
\end{aligned}
$$

Therefore, the sequence $N_{n}^{y}$ is monotonically decreasing and

$$
\lim _{n \rightarrow+\infty} N_{n}^{y}=\frac{\alpha_{1}\left(\alpha_{2} k e^{-d_{11} \tau_{1}}-d_{12}\right)}{\alpha_{1} \beta_{1}+\alpha_{2} c_{1} k e^{-d_{11} \tau_{1}}}=y_{2}^{0} .
$$

Then from (26) and (29) we see that the sequence $N_{n}^{x}$ is decreasing and the sequences $M_{n}^{x}$ and $M_{n}^{y}$ are increasing, furthermore,

$$
\lim _{n \rightarrow+\infty} N_{n}^{x}=x^{0}, \quad \lim _{n \rightarrow+\infty} M_{n}^{x}=x^{0}, \quad \lim _{n \rightarrow+\infty} M_{n}^{y}=y_{2}^{0}
$$

Hence, we obtain

$$
\lim _{t \rightarrow+\infty} x(t)=x^{0}, \quad \lim _{t \rightarrow+\infty} y_{2}(t)=y_{2}^{0}, \quad \lim _{t \rightarrow+\infty} z_{2}(t)=0
$$

Similar to the proof of (22)-(25), by a direct computation, we obtain

$$
\begin{aligned}
& \lim _{t \rightarrow+\infty} y_{1}(t)=\frac{\alpha_{2}}{d_{11}}\left(1-e^{-d_{11} \tau_{1}}\right) x^{0} y_{2}^{0}=y_{1}^{0}, \\
& \lim _{t \rightarrow+\infty} z_{1}(t)=0 .
\end{aligned}
$$

This completes the proof.

In the next, we shall study the global stability of the axial equilibrium $E_{1}(k, 0,0,0,0)$ of system (2) when $k \alpha_{2} e^{-d_{11} \tau_{1}}<d_{12}$.

Theorem 9 The delay due to the maturity of the predator has great impacts on the dynamics for system (2). More precisely, if $\tau_{1} \in\left(m_{2},+\infty\right)$, then the axial equilibrium $E_{1}$ of system (2) is AS. In this case, all predators will go to extinction.

Proof By $\tau_{1} \in\left(m_{2},+\infty\right)$, one finds that $\alpha_{2} k e^{-d_{11} \tau_{1}}<d_{12}$ holds. Using Theorem 4, we find that the axial equilibrium $E_{1}(k, 0,0,0,0)$ is LAS. Hence, it suffices to prove that $\lim _{t \rightarrow+\infty}\left(x(t), y_{1}(t), y_{2}(t), z_{1}(t), z_{2}(t)\right)=(k, 0,0,0,0)$.

The same arguments as those in the proof of Theorem 7 show that (15) holds, i.e.

$$
\limsup _{t \rightarrow+\infty} x(t) \leq k
$$


Hence, for $\varepsilon>0$ sufficiently small, satisfying $\alpha_{2} e^{-d_{11} \tau_{1}}(k+\varepsilon)<d_{12}$, there is a $T_{1}>0$ such that $x(t) \leq k+\varepsilon$ for $t>T_{1}$.

We obtain from the third equation of system (2), for $t>T_{1}+\tau$,

$$
\dot{y}_{2}(t) \leq \alpha_{2} e^{-d_{11} \tau_{1}}(k+\varepsilon) y_{2}\left(t-\tau_{1}\right)-d_{12} y_{2}(t)-\beta_{1} y_{2}^{2}(t)
$$

By applying Lemma 2(ii) and comparison, one can get

$$
\lim _{t \rightarrow+\infty} y_{2}(t)=0
$$

Consequently, for any $\varepsilon>0$ sufficiently small, there exists a $T_{2}>T_{1}+\tau$ such that $0<y_{2}(t)<$ $\varepsilon$ for $t>T_{2}$.

From the first equation of system (2), for $t>T_{2}$,

$$
\dot{x}(t) \geq x(t)\left[\alpha_{1}\left(1-\frac{x(t)}{k}\right)-c_{1} \varepsilon\right] .
$$

Using the comparison theorem,

$$
\liminf _{t \rightarrow+\infty} x(t) \geq \frac{k\left(\alpha_{1}-c_{1} \varepsilon\right)}{\alpha_{1}} .
$$

This inequality holds for $\varepsilon>0$ sufficiently small, one has

$$
\liminf _{t \rightarrow+\infty} x(t) \geq k
$$

By (31) and (32), we obtain

$$
\lim _{t \rightarrow+\infty} x(t)=k
$$

We obtain from the first equation of system (2), for $t>T_{2}$,

$$
\dot{z}_{2}(t) \leq \alpha_{3} e^{-d_{21} \tau_{2}} \varepsilon z_{2}\left(t-\tau_{2}\right)-d_{22} z_{2}(t)-\beta_{2} z_{2}^{2}(t)
$$

By applying Lemma 2(ii) and comparison, one can get

$$
\lim _{t \rightarrow+\infty} z_{2}(t)=0
$$

Similar to the proof of (22)-(25), we obtain $\lim _{t \rightarrow+\infty} y_{1}(t)=0, \lim _{t \rightarrow+\infty} z_{1}(t)=0$.

The proof is complete.

Remark 4 It is obvious that $\alpha_{1} \beta_{1} \beta_{2}>\alpha_{1} \alpha_{3} c_{2} e^{-d_{21} \tau_{2}}+\alpha_{2} \beta_{2} c_{1} k e^{-d_{11} \tau_{1}}$ implies $\alpha_{1} \beta_{1}>$ $\alpha_{2} c_{1} k e^{-d_{11} \tau_{1}}$. And then, by calculating, the condition (C4) can reduce to $\tau_{2}<m_{4}$. Therefore, by Theorem 7 , if the interior equilibrium $E^{*}\left(x^{*}, y_{1}^{*}, y_{2}^{*}, z_{1}^{*}, z_{2}^{*}\right)$ of system (2) is GAS, then the $\tau_{2}$ must satisfy $\tau_{2}<m_{4}$. 
Remark 5 From Theorem 8, when $\tau_{1} \in\left(m_{1}, m_{2}\right)$ and $\tau_{2} \in\left(m_{4},+\infty\right)$, then the boundary equilibrium $E_{2}\left(x^{0}, y_{1}^{0}, y_{2}^{0}, 0,0\right)$ of system (2) is AS, i.e., the prey species and the predator species will coexist, the top-predator species will go extinct. Comparing with Remark 4, one can find that longer delay $\tau_{2}$ will lead the top-predator species to extinction.

Remark 6 According to Theorem 9, when $\tau_{1} \in\left(m_{2},+\infty\right)$, then the axial equilibrium $E_{1}(k, 0,0,0,0)$ of system (2) is AS, i.e., all predators will go extinct. Comparing with the Remark 5 , it is obvious that longer delay $\tau_{1}$ will lead the predators to extinction.

\section{Numerical simulation}

In this section, one example is presented to demonstrate the correctness and effectiveness of the obtained results.

Example 1 Consider the following system with two different time delays:

$$
\left\{\begin{array}{l}
\dot{x}(t)=x(t)\left[15\left(1-\frac{x(t)}{4}\right)-5 y_{2}(t)\right], \\
\dot{y}_{1}(t)=6 x(t) y_{2}(t)-\ln 2 y_{1}(t)-6 e^{-\ln 2 \tau_{1}} x\left(t-\tau_{1}\right) y_{2}\left(t-\tau_{1}\right), \\
\dot{y}_{2}(t)=6 e^{-\ln 2 \tau_{1}} x\left(t-\tau_{1}\right) y_{2}\left(t-\tau_{1}\right)-y_{2}(t)-5 y_{2}^{2}(t)-\frac{1}{4} y_{2}(t) z_{2}(t), \\
\dot{z}_{1}(t)=6 y_{2}(t) z_{2}(t)-\ln 2 z_{1}(t)-6 e^{-\ln 2 \tau_{2}} y_{2}\left(t-\tau_{2}\right) z_{2}\left(t-\tau_{2}\right), \\
\dot{z}_{2}(t)=6 e^{-\ln 2 \tau_{2}} y_{2}\left(t-\tau_{2}\right) z_{2}\left(t-\tau_{2}\right)-z_{2}(t)-2 z_{2}^{2}(t),
\end{array}\right.
$$

where $\tau_{1}>0$ and $\tau_{2}>0$ are constant time delay.

Case I. Let $\tau_{1}=2$ and $\tau_{2}=1$, then

$$
\begin{array}{ll}
\text { (C2) } \quad \alpha_{1} \alpha_{3} c_{2} e^{-d_{21} \tau_{2}}+\beta_{2} c_{1} d_{12}-c_{1} c_{2} d_{22}=20>0 ; \\
\text { (C3) } \alpha_{2} \beta_{2} k e^{-d_{11} \tau_{1}}-\beta_{2} d_{12}+c_{2} d_{22}=10.25>0 ; \\
\text { (C4) } \alpha_{1} \alpha_{3} e^{-d_{21} \tau_{2}}\left(\alpha_{2} k e^{-d_{11} \tau_{1}}-d_{12}\right)-d_{22}\left(\alpha_{1} \beta_{1}+\alpha_{2} c_{1} k e^{-d_{11} \tau_{1}}\right)=120>0 ; \\
& 2 \alpha_{1}-\alpha_{2} k e^{-d_{11} \tau_{1}}=24>0 ; \\
& 2 \beta_{1}>\alpha_{2} e^{-d_{11} \tau_{1}}+\alpha_{3} e^{-d_{21} \tau_{2}}=5.5>0 ; \\
& 2 \beta_{2}>\alpha_{3} e^{-d_{21} \tau_{2}}=1>0, \quad \text { and } \\
& \alpha_{1} \beta_{1} \beta_{2}-\alpha_{1} \alpha_{3} c_{2} e^{-d_{21} \tau_{2}}-\alpha_{2} \beta_{2} c_{1} k e^{-d_{11} \tau_{1}}=78.75>0 .
\end{array}
$$

Thus, the conditions of Theorem 7 hold and the interior equilibrium $E^{*}\left(\frac{526}{177}, \frac{291,141}{31,329 \ln 2}, \frac{123}{177}, \frac{11,808}{10,443 \ln 2}, \frac{32}{59}\right)$ of system (33) is AS. The numerical simulation is shown in Fig. 1.

Case II. Let $\tau_{1}=2$ and $\tau_{2}=10$, then

$$
\begin{aligned}
& \text { (C1) } \quad \alpha_{2} k e^{-d_{11} \tau_{1}}-d_{12}=5>0 ; \\
& \alpha_{3} y_{2}^{0} e^{-d_{21} \tau_{2}}-d_{22}=\frac{30}{7} \times \frac{1}{2^{10}}-1<0 ; \\
& \alpha_{3} e^{-d_{21} \tau_{2}}\left(\alpha_{2} k e^{-d_{11} \tau_{1}}-d_{12}\right)-\beta_{1} d_{22}=5\left(\frac{6}{2^{10}}-1\right)<0, \quad \text { and } \\
& \alpha_{1} \beta_{1}-\alpha_{2} c_{1} k e^{-d_{11} \tau_{1}}=45>0 .
\end{aligned}
$$




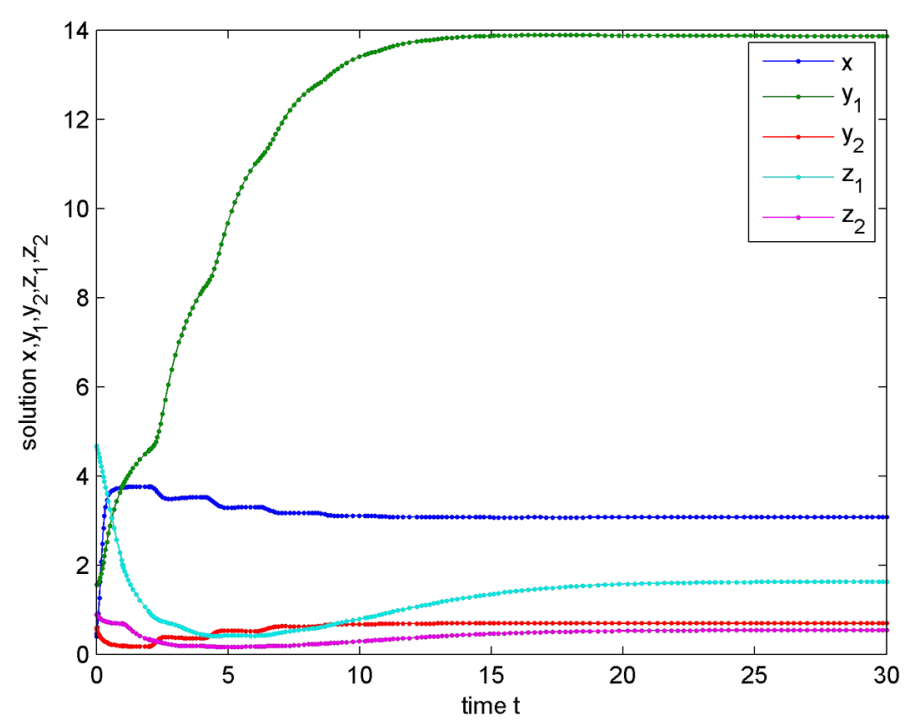

Figure 1 The temporal solution found by numerical integration of system (33) with $\tau_{1}=2, \tau_{2}=1$ and $\left(\phi(\theta), \varphi_{1}(\theta), \varphi_{2}(\theta), \psi_{1}(\theta), \psi_{2}(\theta)\right)=\left(0.4, \frac{27}{25 \ln 2}, 0.6, \frac{81}{50 \ln 2}, 0.9\right)$

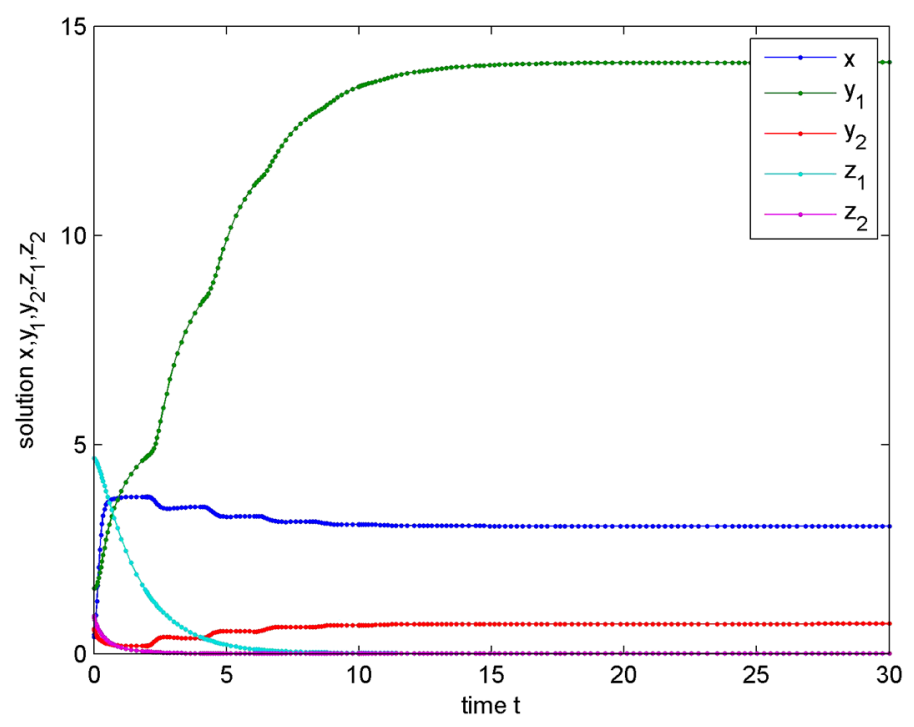

Figure 2 The temporal solution found by numerical integration of system (33) with $\tau_{1}=2, \tau_{2}=10$ and $\left(\phi(\theta), \varphi_{1}(\theta), \varphi_{2}(\theta), \psi_{1}(\theta), \psi_{2}(\theta)\right)=\left(0.4, \frac{27}{25 \ln 2}, 0.6, \frac{81}{25 \ln 2} \times\left(1-\frac{1}{2^{10}}\right), 0.9\right)$

According to Theorem 8 , we can show that the boundary equilibrium $E_{2}\left(\frac{64}{21}, \frac{480}{49 \ln 2}, \frac{5}{7}, 0,0\right)$ of system (33) is AS. The numerical simulation illustrates our result (see Fig. 2).

Case III. Let $\tau_{1}=15$ and $\tau_{2}=1$, then

$$
\alpha_{2} k e^{-d_{11} \tau_{1}}-d_{12}=\frac{3}{2^{12}}-1<0 .
$$

Therefore, the condition of Theorem 9 holds and the axial equilibrium $E_{1}(4,0,0,0,0)$ of system (33) is AS. The numerical simulations also confirm this phenomenon (see Fig. 3). 


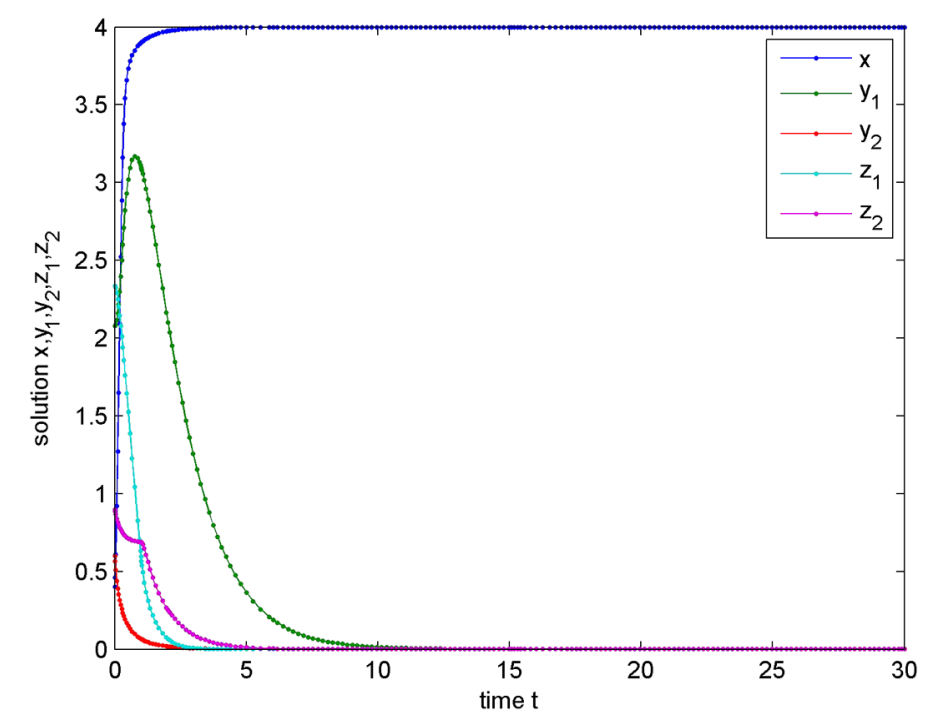

Figure 3 The temporal solution found by numerical integration of system (33) with $\tau_{1}=15, \tau_{2}=1$ and $\left(\phi(\theta), \varphi_{1}(\theta), \varphi_{2}(\theta), \psi_{1}(\theta), \psi_{2}(\theta)\right)=\left(0.4, \frac{36}{25 \ln 2} \times\left(1-\frac{1}{2^{15}}\right), 0.6, \frac{81}{50 \ln 2}, 0.9\right)$

\section{Discussion}

In this paper, by taking full consideration of maturity and stage structure of the predators, a new delayed three-species food-chain model with stage structure for predators is proposed and investigated. The positivity and boundedness of solutions of the model have been verified. By analyzing system (2), the existence and stability of four nonnegative equilibria of system are proved. And ( $\mathrm{C} 1)$ determines the existence of the boundary equilibrium $E_{2} ;(\mathrm{C} 2)-(\mathrm{C} 4)$ determine the existence of the boundary equilibrium $E^{*}$; the trivial equilibrium $E_{0}$ and the axial equilibrium $E_{1}$ exist irrespective of any parameters.

Some interesting findings show that the delays have great impacts on dynamical behaviors for the system: if the delay $\tau_{2}$ is too large, that will account for the top-predator species going to extinction; if the delay $\tau_{1}$ is too large, that will account for the predators to extinction. More precisely, according to Theorems 8 and 9 , if $\tau_{1} \in\left(m_{1}, m_{2}\right)$ and $\tau_{2} \in\left(m_{4},+\infty\right)$, then the prey species and the predator species will coexist, the top-predator species will go extinct; if $\tau_{1} \in\left(m_{2},+\infty\right)$, then all the predators will go extinct.

The obtained results in this paper may provide some new insights for predicting the dynamical behaviors of the food-chain system and protecting the ecological balance in a real ecosystem. By the way, we consider an autonomous system and the coefficient parameters of our model are restricted to constant. However, it would be very challenging whether one can derive sufficient conditions for the dynamical behaviors of the threespecies food-chain model with time-varying coefficients. This will be our future study.

\section{Acknowledgements}

The work is partially supported by the National Natural Science Foundation of China (Nos. 71471020, 11771059); Hunan Provincial Natural Science Foundation of China (No. 2016JJ1001); Scientific Research Fund of Hunan Provincial Education Department (No. 15A003); Hunan Provincial and CSUST Innovation Foundation for Postgraduate (Nos. CX2017B490, CX2017SS23). 
Authors' contributions

All authors read and approved the final manuscript.

\section{Author details}

${ }^{1}$ School of Mathematics and Statistics, Changsha University of Science and Technology, Changsha, P.R. China. ${ }^{2}$ Hunan Key Laboratory of Mathematical Modeling and Analysis in Engineering, Changsha, P.R. China. ${ }^{3}$ Department of Mathematics, Texas A\&M University-Kingsville, Kingsville, USA.

\section{Publisher's Note}

Springer Nature remains neutral with regard to jurisdictional claims in published maps and institutional affiliations.

Received: 11 December 2017 Accepted: 3 April 2018 Published online: 16 May 2018

\section{References}

1. Tripathi, J., Abbas, S., Thakur, M.: Local and global stability analysis of a two prey one predator model with help. Commun. Nonlinear Sci. Numer. Simul. 19, 3284-3297 (2014)

2. Fang, J., Gourley, S., Lou, Y.: Stage-structured models of intra- and inter-specific competition within age classes. J. Differ. Equ. 260, 1918-1953 (2016)

3. Gao, S., Chen, L., Teng, Z.: Hopf bifurcation and global stability for a delayed predator-prey system with stage structure for predator. Appl. Math. Comput. 202, 721-729 (2008)

4. Hu, H., Huang, L.: Stability and Hopf bifurcation in a delayed predator-prey system with stage structure for prey. Nonlinear Anal., Real World Appl. 11, 2757-2769 (2010)

5. Aiello, W., Freedman, H.: A time delay model of single-species growth with stage structure. Math. Biosci. 101, 139-153 (1990)

6. Kuang, Y., So, J.: Analysis of a delayed two-stage population with space-limited recruitment. SIAM J. Appl. Math. 55, 1675-1695 (1995)

7. Wang, W., Mulone, G., Salemi, F., Salone, V.: Permanence and stability of a stage-structured predator-prey model. J. Math. Anal. Appl. 262, 499-528 (2001)

8. Xu, R., Chaplain, M., Davidson, F.: Global stability of Lotka-Volterra type predator-prey model with stage structure and time delay. Appl. Math. Comput. 159, 863-880 (2004)

9. Chen, F., Wang, H., Lin, Y., Chen, W.: Global stability of a stage-structured predator-prey system. Appl. Math. Comput. 223, 45-53 (2013)

10. Kar, T., Jana, S.: Stability and bifurcation analysis of a stage structured predator-prey model with time delay. Appl. Math. Comput. 219, 3779-3792 (2012)

11. Deng, L., Wang, X., Peng, M.: Hopf bifurcation analysis for a ratio-dependent predator-prey system with two delays and stage structure for the predator. Appl. Math. Comput. 231, 214-230 (2014)

12. Xu, C., Yuan, S., Zhang, T.: Global dynamics of a predator-prey model with defense mechanism for prey. Appl. Math. Lett. 62, 42-48 (2016)

13. Ruan, S., Tang, Y., Zhang, W.: Versal unfoldings of predator-prey systems with ratio-dependent functional response. J. Differ. Equ. 249, 1410-1435 (2010)

14. Hu, G., Li, X.: Stability and Hopf bifurcation for a delayed predator-prey model with dsease in the prey. Chaos Solitons Fractals 45, 229-237 (2012)

15. Faria, T., Oliveira, J.: Local and global stability for Lotka-Volterra systems with distributed delays and instantaneous negative feedbacks. J. Differ. Equ. 244, 1049-1079 (2008)

16. Hsu, S., Ruan, S., Yang, T.: Analysis of three species Lotka-Volterra food web models with omnivory. J. Math. Anal. Appl. 426, 659-687 (2015)

17. Paul, P., Ghoshb, B., Kara, T.: Impact of species enrichment and fishing mortality in three species food chain models. Commun. Nonlinear Sci. Numer. Simul. 29, 208-223 (2015)

18. Bologna, M., Chandía, K., Flores, J.: A non-linear mathematical model for a three species ecosystem: hippos in Lake Edward. J. Theor. Biol. 389, 83-87 (2016)

19. Pang, P., Wang, M.: Strategy and stationary pattern in a three-species predator-prey model. J. Differ. Equ. 200 245-273 (2004)

20. Zhou, S., Li, W., Wang, G.: Persistence and global stability of positive periodic solutions of three species food chains with omnivory. J. Math. Anal. Appl. 324, 397-408 (2006)

21. Gupta, R., Chandra, P.: Dynamical properties of a prey-predator-scavenger model with quadratic harvesting. Commun. Nonlinear Sci. Numer. Simul. 49, 202-214 (2017)

22. Shen, C., You, M.: Permanence and extinction of a three-species ratio-dependent food chain model with delay and prey diffusion. Appl. Math. Comput. 217, 1825-1830 (2010)

23. Cui, G., Yan, X.: Stability and bifurcation analysis on a three-species food chain system with two delays. Commun. Nonlinear Sci. Numer. Simul. 16, 3704-3720 (2011)

24. Pei, Y., Guo, M., Li, C.: A delay digestion process with application in a three-species ecosystem. Commun. Nonlinear Sci. Numer. Simul. 16, 4365-4378 (2011)

25. Mbava, W., Mugisha, J., Gonsalves, J.: Prey, predator and super-predator model with disease in the super-predator. Appl. Math. Comput. 297, 92-114 (2017)

26. Huang, C., Yang, Z., Yi, T., Zou, X.: On the basins of attraction for a class of delay differential equations with non-monotone bistable nonlinearities. J. Differ. Equ. 256, 2101-2114 (2014)

27. Huang, C., Cao, J., Xiao, M., Alsaedi, A., Alsaadi, F.: Controlling bifurcation in a delayed fractional predator-prey system with incommensurate orders. Appl. Math. Comput. 293, 293-310 (2017)

28. Huang, C., Gong, X., Chen, X., Wen, F.: Measuring and forecasting volatility in Chinese stock market using HAR-CJ-M model. Abstr. Appl. Anal. 2013, 1 (2013) 
29. Huang, C., Peng, C., Chen, X., Wen, F.: Dynamics analysis of a class of delayed economic model. Abstr. Appl. Anal. 2013, 1 (2013)

30. Wang, Y., Cao, J.: Global dynamics of a network epidemic model for waterborne diseases spread. Appl. Math. Comput. $237,474-488(2014)$

31. Huang, C., Cao, J.: Convergence dynamics of stochastic Cohen-Grossberg neural networks with unbounded distributed delays. IEEE Trans. Neural Netw. 22, 561-572 (2011)

32. Huang, C., Cao, J., Xiao, M., Alsaedi, A., Hayat, T.: Effects of time delays on stability and Hopf bifurcation in a fractional ring-structured network with arbitrary neurons. Commun. Nonlinear Sci. Numer. Simul. 57, 1-13 (2018)

33. Huang, C., Cao, J.: Impact of leakage delay on bifurcation in high-order fractional BAM neural networks. Neural Netw. 98, 223-235 (2018)

34. Huang, C., Cao, J., Cao, J.: Stability analysis of switched cellular neural networks: a mode-dependent average dwell time approach. Neural Netw. 82, 84-99 (2016)

35. Song, X., Chen, L.: Optimal harvesting and stability for a two-speices competitive system with stage structure. Math. Biosci. 170, 173-186 (2001)

Submit your manuscript to a SpringerOpen ${ }^{\circ}$ journal and benefit from:

- Convenient online submission

- Rigorous peer review

- Open access: articles freely available online

- High visibility within the field

- Retaining the copyright to your article

Submit your next manuscript at $\gg$ springeropen.com 